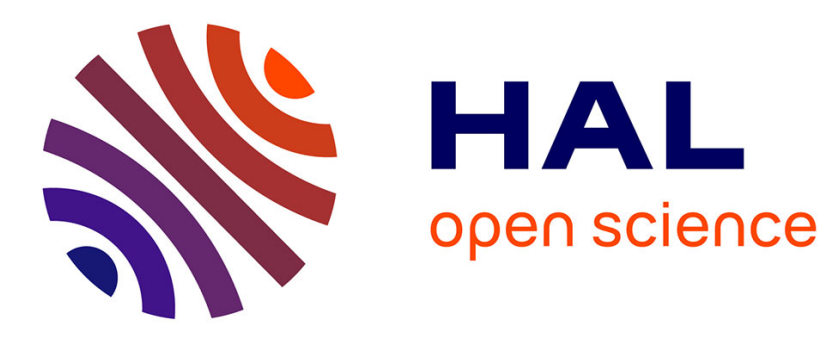

\title{
On reversals in 2D turbulent Rayleigh-Bénard convection: insights from embedding theory and comparison with Proper Orthogonal Decomposition analysis
}

\author{
Davide Faranda, Bérengère Podvin, Anne Sergent
}

\section{To cite this version:}

Davide Faranda, Bérengère Podvin, Anne Sergent. On reversals in 2D turbulent Rayleigh-Bénard convection: insights from embedding theory and comparison with Proper Orthogonal Decomposition analysis. Chaos: An Interdisciplinary Journal of Nonlinear Science, 2019, 29 (29), pp.033110. 10.1063/1.5081031 . hal-01701353v2

\section{HAL Id: hal-01701353 \\ https://hal.science/hal-01701353v2}

Submitted on 6 Feb 2019

HAL is a multi-disciplinary open access archive for the deposit and dissemination of scientific research documents, whether they are published or not. The documents may come from teaching and research institutions in France or abroad, or from public or private research centers.
L'archive ouverte pluridisciplinaire HAL, est destinée au dépôt et à la diffusion de documents scientifiques de niveau recherche, publiés ou non, émanant des établissements d'enseignement et de recherche français ou étrangers, des laboratoires publics ou privés. 


\section{On reversals in 2D turbulent Rayleigh-Bénard convection: insights from embedding theory and comparison with Proper Orthogonal Decomposition analysis}

D. Farandat

LSCE-IPSL, CEA Saclay l'Orme des Merisiers, CNRS UMR 8212 CEA-CNRS-UVSQ, Université Paris-Saclay, 91191 Gif-sur-Yvette, Franc®

B. Podvin

LIMSI, CNRS, Université Paris-Saclay, Campus Universitaire, 91405 Orsay, France

A. Sergent

LIMSI, CNRS, Université Paris-Saclay, Campus Universitaire, 91405 Orsay, France, Sorbonne Université, Faculté des Sciences et Ingénierie, UFR d'Ingénierie, 75005 Paris, France

Turbulent Rayleigh-Bénard convection in a 2D square cell is characterized by the existence of a large-scale circulation which varies intermittently. We focus on a range of Rayleigh numbers where the large-scale circulation experiences rapid non-trivial reversals from one quasi-steady (or metastable) state to another. In previous work (Podvin and Sergent JFM 2015, Podvin and Sergent PRE 2017), we applied Proper Orthogonal Decomposition (POD) to the joint temperature and velocity fields at a given Rayleigh number and the dynamics of the flow were characterized in a multi-dimensional POD space. Here, we show that several of those findings, which required extensive data processing over a wide range of both spatial and temporal scales, can be reproduced, and possibly extended, by application of embedding theory to a single time series of the global angular momentum, which is equivalent here to the most energetic POD mode. Specifically, embedding theory confirms that the switches among meta-stable states are uncorrelated. It also shows that, despite the large number of degrees of freedom of the turbulent Rayleigh Benard flow, a low dimensional description of its physics can be derived with low computational efforts, providing that a single global observable reflecting the symmetry of the system is identified. A strong connection between the local stability properties of the reconstructed attractor and the characteristics of the reversals can also be established. 
We use Rayleigh-Bénard turbulent convection simulations to compare the properties of quasistationary states and transitions (reversals) previously identified via Principal Orthogonal Decomposition (POD) to those obtained via embedding the global angular momentum of the system. Our main result is to show that we can map POD properties on the attractor reconstructed via embedding techniques. Specifically, the embedding technique confirms that the switches among different metastable states are uncorrelated. Moreover, a local stability indicator can be used to distinguish and classify the different metastable states. The low computational costs of embedding analysis suggests to use this procedure whenever a global observable reflecting the symmetry of the system can be identified, while the POD should be preferred when such information is not available.

\section{INTRODUCTION}

In the last decades, the study of complex systems has shifted from a pure dynamical systems-based approach to a mixture of statistical and statistical-mechanics techniques. This can be justified on both practical and theoretical levels. At the beginning of the 80's, the abundance of data from measurements as well as from numerical models was on a control parameter [1 2. The use of numerical weather forecasts was limited to a few days [3] and climate models were mostly conceptual [4]. At that time, new results in dynamical systems theory seemed to convince the scientific community that a sufficiently long time series could be sufficient to reconstruct the dynamics of the system via its attractor. This object is a geometric set towards which the system tends to evolve, independently on its initial conditions.

The Takens [5, 6] reconstruction theorem was then used to determine the dynamics of climate attractors as well as of complex flows. Initially, the dimension of these objects was set to be extremely low, usually smaller than 10 [7. However, from the beginning of the 90's, several authors [8 10] found out that those low dimensional estimates were wrong: in fact, despite the complexity of the systems, the reconstructions were mostly made using time series pasule at specife points of the physical space. Moreover, the time series used were not sufficity long. In parallel, computational power increased quickly [11] and measurement techniques opened new possibilities to sample the behavior of complex systems [12. In experimental facilities, visualization techniques gave rapid access to field measurements instead of local measurements[13, 14 and numerical models were capable to simulate several years of Earth's climate[15], seconds of human brain activity[16, and molecules/proteins dynamics [17, 18].

The complexity of high-dimensional fields as opposed to single-point time series required new methodologies in order to assess the probability of each configuration and forecast the evolution of the system. For this purpose, one of the most popular techniques is the Proper Orthogonal Decomposition (POD). In POD, an orthogonal transformation is used to transform a dataset with correlated variables into linearly uncorrelated variables. Geometrically, this is like fitting an $n$-dimensional ellipsoid to the data, where each axis of the ellipsoid represents a principal component. 6o Principal Component Analysis was invented by Karl Pearson[19], as an analogue of the principal axis theorem in mechanics. In the 1930s it was developed independently also by Harold Hotelling [20]. Depending on the field of application, it is also named the discrete Kosambi-Karhunen-Loève transform (KLT) in signal processing [21, the Hotelling transform [20] in multivariate quality control, proper orthogonal decomposition (POD) in turbulence [22, empirical orthogonal functions (EOF) in meteorological science [23, empirical eigenfunction decomposition [24, and it is also connected to singular value decomposition (SVD) of X [25], eigenvalue decomposition (EVD) of XTX in linear algebra, factor analysis, Eckart-Young theorem [26, or Schmidt-Mirsky theorem in psychometrics, quasiharmonic modes [27, spectral decomposition in noise and vibration, and empirical modal analysis in structural dynamics [28]. The belief that embedding theorems could not be applied either to systems featuring a large number of degrees of freedom or to time series of high-dimensional vector fields, caused the data analysis scientific community to focus on POD-based approaches to study complex systems. Meanwhile, several theoretical developments in dynamical systems theory were aimed at understanding how systems with large numbers of degrees of freedom could be dealt with [29, 30]. In particular, the framework of stochastic dynamical systems [31, 32] brings into view the idea that the dynamics of complex systems can be represented by a small number of variables if one lumps the small scales contributions into noise terms and choose as observables for the embedding procedure, global quantities tracking symmetry properties 75 of the flow.

For turbulent flows, such as the von Karman swirling flow, or for atmospheric dynamics, this revised approach produces phase portraits which make it possible to define low-dimensional models which capture the essential features of the dynamics [33] in the fashion of the 1963 Lorenz equations [34. The goal of the present paper is to examine how information provided by the embedding procedure compares with that obtained by a more extensive analysis such as 
so the Proper Orthogonal Decomposition (POD). This will be done for the specific case of 2-D turbulent Rayleigh-Bénard convection, a flow which is characterized by a series of random transitions from a quasi-steady state to another. This system is an interesting platform for exploring the relation between POD and embedding methods for few reasons: i) It is a turbulent flow and this would prevent - at least according Landau's conjecture [35] - a projection of the dynamics onto a low dimensional space due to the large number of degrees of freedom introduced by small scales perturbations. ii) The dynamics features a non-trivial bifurcation structure. This is only observed in a limited range of Prandtl and Rayleigh numbers, for reasons that are not understood [36]. iii) The dynamics for some values of the parameter analysed in the paper feature the switching among different metastable states, plus reversal with some hybrid states. The physical origin of the switching remains largely unknown.

The paper is structured as follows: first we will recap the methodologies used, with emphasis on the recent developments on the embedding strategies for complex systems. Then we will describe the dataset used and outline the results. Eventually we will discuss the advantage of a combined approach to complex systems analysis.

\section{DYNAMICAL SYSTEMS METHODS}

In this section we give some elements of dynamical systems theory and some guidelines derived from recent studies for reconstructing the attractors using global rather than local observables. We also introduce indicators of stability derived from the theory of stochastic processes.

\section{Attractor Reconstruction via embedding methodologies}

The aim of dynamical systems theory is to reconstruct the underlying attractor of a system and to assess its properties. The attractor is a compact geometrical object in phase space that hosts all the possible trajectories of a system. Once this object is reconstructed, it provides information on the (unstable) fixed points (if the system is deterministic) or the metastable states (if the system is stochastic) and the transitions linking them. This description is useful when the attractor is low dimensional because its structure can be visualized and interpreted in an intuitive way. Up to recently it was believed that low dimensional attractors would not be suitable to describe complex geophysical and turbulent flow. However, it has beeen shown [33, that it is possible to describe the large scale motion of a fully-developed turbulent flow with only a few degrees of freedom, if an appropriate observable reflecting the theory to turbulence arise from small scale disturbances that can be modeled in terms of stochastic perturbations. This picture reconciles the Landau [35] and Ruelle-Takens [37] descriptions of turbulence, the former being valid at small scales, and the latter describing the large scale motions. The observable can be derived as global mean of some local quantities in the flow (average energy or momentum), or it can be a single global output quantity measured in an experiment (such as for instance the frequency of rotation of Von Karman flow turbines, once a certain torque is imposed).

Once the observable is selected and a time series is obtained, different embedding procedures can be used to reconstruct the attractor from the signal. The first thing to determine is the embedding dimension, i.e. the number of variables necessary for the attractor reconstruction. This can be done using the method by Cao [38, or by trying to see how much information is added using another dimension. In what follows we will adopt the second strategy. After selecting the number of variables $n$, embedding consists in defining a n-dimensional state vector from the observable time series $X_{i}$ consisting $i=1,2, \ldots t$ observations, as $M_{i}=\left(X_{i}, X_{i+\tau_{1}}, X_{i+\tau_{2}}, \ldots\right)$. The series can be embedded with the method of delays [5], or with the local peaks procedure [39]. In the method of delays, the value of $\tau_{i}$ is kept constant, which is most effective when there is a precise time scale in the system. In contrast, the local peaks method consists in selecting $\tau_{i}$ dynamically as the interval between two subsequent partial maxima (or minima), which is most effective when there are no well-identified time scales (e.g. switching frequencies between metastable states). In fact, local maxima (or minima) are robust features of the dynamics even in the presence of noise, because they track the position of metastable states [33. Since we deal with a turbulent system, we stick to the local peaks methodology and we refer the reader to $[33$ for a comparison of the two methods.

Once the series of $m$ partial maxima is obtained, the attractor is visualized by plotting in a $n$-dimensional phase space, $M_{j}, M_{j+1}, \ldots, M_{j+n}$, where $j=1,2, \ldots, m$. As mentioned earlier, the value of $n$, known as the embedding dimension, plays a crucial role in the applications of dynamical systems theory to real data [40]. 


\section{Stability indicator}

To characterize the stability of the attractor, we will use the indicator $\Upsilon$ introduced in [41]. We define our stability indicator by using a simple example: even for a complex system, the dynamics near a metastable state resembles that of a stochastic spring (or of a particle in a quadratic potential). The typical equation associated to these systems is the Langevin equation:

$$
\frac{\mathrm{d} X(t)}{\mathrm{d} t}=-\frac{k}{m} X(t)+\frac{1}{m} \xi(t)
$$

where $k$ is a frictional force (e.g. the Stokes' drag), $m$ is the mass of the particle and $\xi$ is a noise term modeling the random collisions the particle undergoes. The discretized equation then becomes:

$$
X_{t}=\phi X_{t-1}+\epsilon_{t}
$$

This defines a linear process called an ARMA, an autoregressive moving-average model. These models have been widely used over the past decades, especially in econometrics and finance to forecast markets trends. An ARMA model is characterized by $p$ autoregressive terms and $q$ moving-average terms and is denoted $\operatorname{ARMA}(p, q)$. The equation above is therefore an $\operatorname{ARMA}(1,0)$. We briefly present the normal form of an $\operatorname{ARMA}(p, q)$ model and some criteria used to fit it to a time series (see 42 for a detailed review).

Let us consider a series $X(t)$ of an observable with unknown underlying dynamics. We further assume that for a time scale $\tau$ of interest, the time series $X_{t_{1}}, X_{t_{2}}, \ldots, X_{t \tau}$ represents a stationary phenomenon. Since $X_{t}$ is stationary, we may then model it by an $\operatorname{ARMA}(\mathrm{p}, \mathrm{q})$ process such that for all $t$ :

$$
X_{t}=\sum_{i=1}^{p} \phi_{i} X_{t-i}+\varepsilon_{t}+\sum_{j=1}^{q} \theta_{j} \varepsilon_{t-j}
$$

with $\varepsilon_{t} \sim W N\left(0, \sigma^{2}\right)$ - where $W N$ stands for white noise - and the polynomials $\phi(z)=1-\phi_{1} z_{t-1}-\ldots-\phi_{p} z_{t-p}$ $\theta(z)=1-\theta_{1} z_{t-1}-\ldots-\theta_{q} z_{t-q}$, with $z \in \mathbb{C}$, have no common factors. Notice that, hereinafter, the noise term $\varepsilon_{t}$ is assumed to be a white noise. For a general stationary time series, this model is not unique. However there are several standard procedures for selecting the model which best fits the data. The one we exploit is the Bayesian information criteria [43]. It is based on the Akaike information criteria (AIC) [44] which was designed to be an approximately unbiased estimate of the Kullback-Leibler index of the fitted model relative to the true model. Assuming we know the likelihood estimators $\beta$ and $\sigma^{2}$ of the fitted model model thanks to an innovation algorithm, the best ARMA model is the one where $p$ and $q$ minimize

$$
\operatorname{AIC}(\beta)=-2 \ln L_{X}\left(\beta, \sigma^{2}\right)+2(p+q+1)
$$

In order to correct the tendency of the AIC to prefer complex models, we use the BIC (Bayesian information criteria) which introduces a penalty for large-order models:

$$
\mathrm{BIC}=(\tau-p-q) \ln \left(\frac{\tau \sigma^{2}}{\tau-p-q}\right)+\tau(1+\ln \sqrt{2 \pi})+(p+q) \ln \left(\left(\sum_{t=1}^{\tau} X_{t}^{2}-\tau \sigma^{2}\right) /(p+q)\right)
$$

Intuitively, $p$ and $q$ are related to the memory lag of the process, while the coefficients $\phi_{i}$ and $\theta_{i}$ represent the persistence: the higher their sum (in absolute value), the slower the system forgets its past history, the higher the correlations in the time series. Most of the time ARMA models are used in econometrics fitting the whole time series and trying to forecast the future trend of the variable. This assumes a correlation with the past and provide some significant results for the very near future. Our interest here is rather to use ARMA to detect the local stability. The procedure is the same as for fitting the whole time series: after slicing the time series by intervals $\tau$, we obtain a time series $X_{t-\tau}, \ldots, X_{t-1}$. We then fit each $\operatorname{ARMA}(p, q)$ model until $p \leq p_{\max }$ and $q \leq q_{\max }$ assessing the best one (according the BIC criterion). We then compute the stability indicator $\Upsilon$ for the system at time $t$ and then move to time $t+1$ to perform the same analysis on the time series $X_{t-\tau+1}, \ldots, X_{t}$.

When the system is close to an unstable point, separating multiple basins of attraction, the behavior cannot be described by a Langevin equation as the underlying potential is not quadratic anymore. The change in the shape of 
the potential introduces new correlation in the time series resulting in higher order ARMA terms. The indicator is then defined as:

$$
\Upsilon=1-\exp \left[-\frac{|\mathrm{BIC}(p, q)-\mathrm{BIC}(1,0)|}{\tau}\right]
$$

Thus, $\Upsilon$ gives us a normalized distance between the stablest state the particle could be in $(\Upsilon=0)$ and the state where it really is. The limit $\Upsilon \longrightarrow 1$ correspond to a very unstable state, where the particle is a the edge of a basin of attraction and the probability to jump to another connected basin is high.

The only free parameter is the choice of $\tau$. To understand its role, we revert to the spring example: the characteristic time scale of the problem is the relaxation time of the particle to the basin of attraction. This defines a typical time scale of the system. The $\Delta t$ between subsequent observation of the time series should be close to this quantity. Instead, $\tau$ must be a multiple of this quantity but should be smaller than the residence time in the basin of attraction. In previous works, some of the authors of this paper have shown the validity of the $\Upsilon$ indicator to study financial [41] and climate time series 45 .

\section{POD ANALYSIS}

The goal of the paper is to examine how embedding theory results compare with those from a data-intensive analysis technique such as Proper Orthogonal Decomposition (POD). Proper Orthogonal Decomposition extracts the most energetic spatial or temporal flow patterns from either time series or a collection of spatial fields such as the velocity and/or the temperature. Any physical field $q(\underline{x}, t)$ can be decomposed into the superposition of an infinity of spatial structures $\underline{\phi}^{n}(\underline{x})$, the amplitude of which, $a^{n}(t)$, varies in time:

$$
\underline{q}(\underline{x}, t)=\sum_{n=1}^{\infty} a^{n}(t) \underline{\phi}^{n}(\underline{x})
$$

where the $\underline{\phi}^{n}(x)$ are eigensolutions of the eigenvalue problem

$$
\int R\left(\underline{x}, \underline{x}^{\prime}\right) \cdot \underline{\phi}\left(\underline{x}^{\prime}\right) d \underline{x}^{\prime}=\lambda^{n} \underline{\phi}(\underline{x})
$$

where $R\left(\underline{x}, \underline{x}^{\prime}\right)$ is the spatial autocorrelation tensor at zero time lag. The associated eigenvalues $\lambda^{n}=<\left(a^{n}\right)^{2}>$, where $<>$ represents a temporal average, correspond to the energy level of the $n$-th spatial eigenfunction. By construction, the amplitudes $a^{n}$ are uncorrelated and the spatial eigenfunctions orthogonal. Since the field at a given time can be expressed by the instantaneous amplitudes $\left\{a^{n}(t)\right\}$ of the corresponding spatial eigenfunctions, POD directly provides a dynamical systems representation of the flow in a natural manner. The representation is energetically optimal, to the extent that the first N POD modes capture on average more energy than any other basis of size N [46].

The spatial eigenfunctions are extracted from the second-order statistics of $q$, so that Proper Orthogonal Decomposition requires an extensive knowledge of the fully resolved flow and acts as a powerful data reduction technique. It is therefore of interest to compare results from this approach with the embedding technique, which is based on limited data.

\section{RAYLEIGH-BÉNARD CONVECTION}

In this section we provide a description of the flow physics. The configuration consists of a Rayleigh-Bénard square (2-D) cell in the turbulent regime (see figure 1). The top and the bottom plate of the cell are maintained at different temperatures, with a hotter bottom plate and colder top one. This leads to the generation of temperature plumes along the plates, which detach from the boundary layer and are transported into the flow. Natural flow in the square cell is characterized by two parameters. One is the Prandtl number $\operatorname{Pr}=\nu / \kappa$ where $\nu$ is the kinematic viscosity and $\kappa$ the thermal diffusivity. Here we consider water $(\operatorname{Pr}=4.3)$. The other is the Rayleigh number $R a=\frac{\alpha g \Delta T H^{3}}{\nu \kappa}$ which

measures the ratio of buoyancy and diffusive effects, with $\alpha$ the thermal expansion coefficient, $g$ the gravity, $\Delta T$ the temperature difference between the plates, $H$ the dimension of the cell. Adiabatic boundary conditions are imposed on the cell sides. 
The flow is numerically simulated in the Boussinesq approximation using a spectral code (see [47 for details). The simulation parameters are given in table I] The equations are made nondimensional using as characteristic units the cell height $H$ for length, the temperature difference $\Delta T$ for temperature and the convective velocity $R a^{1 / 2} \kappa / H$ for velocity. More details on the numerical simulation can be found in [48. Three different Rayleigh numbers $R a=3 \cdot 10^{7}$, $R a=5 \cdot 10^{7}$ and $R a=10^{8}$ are considered in the present study. For each of these Rayleigh numbers, the small-scale plumes are advected by a large-scale circulation (or roll), which can take two different orientations (see figure 1). The orientation of the roll remains nearly constant for a large portion of the time, but may switch randomly from one state to another over a relatively short duration (transition or reversal). Figure 1 shows two typical flow realizations at $R a=5 \cdot 10^{7}$ respectively preceding and following a reversal. The flow therefore displays random large-scale pattern changes in the presence of small-scale fluctuations. The occurrence and frequency of reversals depends on the Rayleigh number and the Prandtl number as shown by Sugiyama et al [36]. Reversal processes in 2-D or near 2-D cells have been the object of several investigations (Sugiyama et al. [36], Chandra et al., [49], Ni et al. [50]), while several models have also been derived for reversals occuring in a cylinder cell (Brown and Ahlers [51, Sreenivasan et al. Araujo et al. [52, 53, Benzi [54]). More recently, Podvin and Sergent [48, [55, have used Proper Orthogonal Decomposition (POD) to construct dynamical systems to reproduce reversals in the square $2-\mathrm{D}$ cell at $R a=510^{7}$. By use of a time rescaling of the temporal series, Castillo et al. [56] have evidenced a generic reversal cycle consisting of three successive phases.

\section{RESULTS}

\section{POD spectrum}

In previous works [48, [55], we have used POD to investigate the large-scale structure of the flow at $R a=5 \cdot 10^{7}$. POD was applied to the joint temperature and velocity fields, which amounts to defining a POD "energy", corresponding to the sum of the kinetic energy and thermal energy in the nondimensional units defined in the previous section [48. In this work we show results for two other Rayleigh numbers: $R a=3 \cdot 10^{7}$ and $R a=10^{8}$. The number of snapshots and separation between snapshots used in the study is given in table [1. We checked that at all Rayleigh numbers the first four modes corresponded to the same spatial structures described in 555. The amplitude of the dominant mode $a^{1}$, which corresponds to a large-scale circulation scaling with the cell size, is shown in Figure 2 It is shown that reversals are more frequent as $R a$ increases. Moreover the amplitude $a^{1}$ is almost entirely correlated with the global angular momentum $L$ (with a correlation coefficient larger than 0.95). This confirms that the choice of the global angular momentum $L$, which is directly proportional to $a^{1}$, is relevant to carry out the analysis. In the remainder of the paper we will speak indifferently of $L$ or $a^{1}$ (we actually use the notation $L$ for $a^{1}$ in [48] and [55]). We note that this is a very particular case as in general the chosen physical indicator may not coincide with a single POD mode. However it is reasonable to expect that a suitable global indicator could be well approximated with a limited combination of POD modes, so that the observations made here should remain relevant in the general case.

The first four modes of the POD capture more than $85 \%$ of the total POD energy. The full eigenvalue spectrum is represented in Figure 3 and shows that the energy in the first modes is similar for $R a=5 \cdot 10^{7}$ and $R a=10^{8}$ and larger than at $R a=3 \cdot 10^{7}$. igure 3 (right) shows that the third mode is the least important for $R a=3 \cdot 10^{7}$, which can also be seen in table III It is eight times less energetic than for the two higher Rayleigh numbers. However, as can be seen in table III] the different behaviors for different Rayleigh numbers do not correspond to a global increase in the energy of small scales. This suggests that the increase of the reversal frequency is associated with a higher energy in the lowest order modes, and in particular mode 3.

\section{Phase portraits}

The flow dynamics can be characterized by the phase portraits of the POD amplitudes given in Figures 4 to 6 (the amplitudes in the figure $a_{N}^{i}=a^{i} /\left(\lambda^{i}\right)^{1 / 2}$ are normalized). As expected, the system spends a large portion of the time near one of two quasi-stable states (corresponding to a large-scale circulation which can be oriented either clockwise or counterclockwise, as was evidenced in figure 1). Each of the quasi-stable states can be defined in the POD space as $a_{e q}^{ \pm}=\left\{ \pm\left|a_{e q}^{1}\right|,\left|a_{e q}^{2}\right|, 0,-\left( \pm\left|a_{e q}^{4}\right|\right)\right\}$. At random times it makes an excursion far from the state. The excursion is generally brief, and typically ends up with the system approaching the opposite steady state, which constitutes a reversal. Again, despite similarities, differences between the Rayleigh numbers are clear in the phase portraits. As the Rayleigh number increases, the second mode takes smaller positive and even occasionally negative values during 
excursions (Figure 4). The system appears to spend more time outside the quasi-stable regions for the two highest Rayleigh numbers. At $R a=5 \cdot 10^{7}$, the system appears to spend a good portion of that time near $a^{1}=0$, which corresponds to a state where the large-scale circulation has disappeared. This is less present at $R a=10^{8}$, where the presence of a limit cycle appears most probable, as can be seen in particular by comparing the different phase portraits in the $\left(a^{1}, a^{4}\right)$ space.

The phase portraits can be compared with the embedding portraits represented for three different $\Delta t$ in Figure 7 . The dynamics consists of two main fixed points, symmetric and located at $|L| \simeq 0.07$, and two marginal fixed points at $|L| \simeq 0.02$. They are connected by two limit cycles. By increasing the Rayleigh number it is evident that the stability of the fixed points decreases and the dynamics consist of limit cycles rather than fixed points. One can see that both limit cycles and fixed points are identified with the POD representation (Figures 4 to 6). While at $R a=3 \cdot 10^{7}$, the two main fixed points are identifiable, their structure becomes more stretched and circular as $R a$ increases. It is interesting to relate these findings to the predictions reported in [48] and [55] for two models derived from the POD at $R a=5 \cdot 10^{7}$ with respective dimensions of 3 and 5 . The dynamics of the three-dimensional model was characterized (with two extra modes) displayed a limit cycle (55]).

In the embedding method, we find that the optimal embedding dimension for the attractor is 2 , which implies that reversals from one state to another are independent from each other. This is an important result, which is in agreement with experimental measures of the inter-reversal period, which was found to be well modelled by a Poisson distribution in cylinder cells by Xi and Xia [57 and Brown et al. [51. This is in contrast with the results found for the Von Karman flow [33. For this flow, the attractor is 3D which suggests that subsequent reversals are not independent. A possible explanation of this difference is the role of the hysteresis observed in the von Karman flow.

\section{Stability analysis}

We compute the stability parameter $\Upsilon$ using the following procedure: i) we coarse grain the time series of $L$ at the different $\Delta t=3,6,9$ used for the attractor reconstruction, ii) for each of the value $L(t)$ obtained, we used the time series $L(t-\tau), L(t+\Delta t-\tau), \ldots L(t)$ where $t \geq \tau$ to compute $\Upsilon$. Note that we fix $\tau=50$ for the statistical needs outlined in [41].

The values of $\Upsilon$ for each point of the time series and different $\Delta t$ are reported in Figures 8 10. The histogram of $\Upsilon$ and the bivariate histogram $L, \Upsilon$ are reported in figure 11. Overall, we remark that there is a general agreement among the results obtained at different $\Delta t$. For $R a=3 \cdot 10^{7}$, most of the values of $\Upsilon$ are close to 0 , as the dynamics is that of a noisy fixed points. During the reversal and immediately after the values of $\Upsilon$ are higher, indicating the switching of the dynamics. This is why $\Upsilon$ histograms (Figure 11 left) show a principal mode for low value of $\Upsilon$ and a small mode at higher values. The dynamics is different for the cases $R a=5 \cdot 10^{7}$ and $R a=110^{8}$ as for the presence of the limit cycle. The periodicity of the reversals and the sporadic presence of a noisy fixed points dynamics leads to a general increase of $\Upsilon$ values.

The theory says that $\Upsilon$ should be large when rapid changes occur in the dynamics, i.e at the onset or at the end of reversals. This means that large values of $\Upsilon$ should coincide with the edges of the attractor. Interestingly, as the Rayleigh number increases, large values of $\mathrm{Y}$ are found for an increasing and continuous range of $L$ values, which is not easy to understand with a purely one-dimensional representation. Indeed, the histogram of $a^{1}(\mathrm{~L})$, shown in figure 12, does not provide a clear picture of the attractor. In particular, there does not seem to be a marked difference between $R a=10^{8}$ and $R a=5 \cdot 10^{7}$. However, multi-dimensional histograms in the POD space, such as the joint histogram in the $a^{1}-a^{2}$ plane shown in figure 13 , show that the attractor is elongated in the $a^{1}$ direction for increasing Rayleigh numbers. The values of $a^{1}$ associated with the edge of these regions agree well with the values of $L$ for which $\Upsilon$ is high. Lower values of $L\left(a^{1}\right)$ over a relatively small extent are associated with the case $R a=3 \cdot 10^{7}$, while the values increase over a wider range for $R a=5 \cdot 10^{7}$ and even more so for the case $R a=10^{8}$.

\section{Time between reversals}

A characteristic of the flow reversals can be given by the time intervals $T$ between zeros of the dominant amplitude $a^{1}$. Histograms of the time intervals are represented in figure $\left.14 \mathrm{a}, \mathrm{b}, \mathrm{c}\right)$. The mean values of the separation times $\langle T\rangle$, given in table III are very similar at the two highest Rayleigh numbers, while the mean value is higher by a factor of 6 in the case $R a=3 \cdot 10^{7}$. To try and understand this from a dynamical systems perspective, let us consider model 
dynamics consisting of a heteroclinic cycle characterized by a single unstable eigenvalue $\sigma u$ and submitted to a noise of amplitude $\epsilon$. In that case Holmes et al. [46] have shown that a mean period $T_{H C}$ can be defined for the heteroclinic cycle, where $T_{H C}$ is the average time the system needs to leave the attractor, move towards the other attractor and come back again (so with our notations we have $T=\frac{1}{2} T_{H C}$ ). This mean period can be expressed as

$$
\left.T_{H C} \sim 2\left(K_{0}+\frac{1}{\sigma u}\left(K_{1}+\log \left(\frac{1}{\epsilon}\right)\right)\right)\right)
$$

where $K_{0}$ and $K_{1}$ are constants.

A crude estimate of the noise level here can be given by the standard deviation of the distance $d_{e q}$ between the current vector state $a^{i}, 1 \leq i \leq 4$ and the nearest quasi-steady states $a_{e q}^{ \pm}$:

$$
d_{e q}=\min \left(\left\|a-a_{e q}^{+}\right\|,\left\|a-a_{e q}^{-}\right\|\right)
$$

The estimate for the noise level $d_{e q}^{r m s}$ is reported in table III At $R a=5 \cdot 10^{7}$ and $R a=10^{8}$, the noise level appears similar and the mean reversal rate $1 /\langle T\rangle$ is about the same, which suggests that the unstable growth rate is also the same, which is consistent with similar mean values of $\Upsilon\left(0.59\right.$ for $R a=10^{8}$ and 0.56 for $\left.R a=5 \cdot 10^{7}\right)$. In contrast, $\Upsilon$ is on average twice as small at $R a=3 \cdot 10^{7}$ its value being 0.26 , while the average reversal rate is six times smaller. However this difference is consistent with the fact that the noise level is smaller by a factor of 4 at this Rayleigh number.

We now take a detailed look at the distribution of the times normalized by their time-averaged value, which is shown in Figure 14. Normalizing the time separation $T$ by its mean value $\langle T\rangle$ can be seen as loosely equivalent to the normalization used in the definition of the stability indicator. Although strict equivalence is not possible, since the indicator varies only between 0 and 1 , we have also represented in the middle row of figure 14 the distribution of the normalized separation times limited to the interval $[0,1]$. It is of interest to compare the histograms of $T /\langle T\rangle$, which are obtained from the full time-series and over long periods of time, with the distributions of the indicator $\Upsilon$ shown in Figure 11, which is extracted from application of the autoregressive model over a short period of time. The general shape of the time distribution is different for each Rayleigh number. The very low values of the separation times observed at all Rayleigh numbers correspond to times which are smaller than a full transition time (i.e a complete switch from one metastable state to another). This means that there is no real reversal corresponding to corresponds to the temporary disappearance of a large-scale circulation (which is termed a cessation). The low values are frequent at $R a=3 \cdot 10^{7}$ and $R a=5 \cdot 10^{7}$, but less so at $R a=10^{8}$, in agreement with both embedding and POD phase portraits. The low reversal rate at $R a=310^{7}$ is consistent with a mildly unstable system, which agrees with the distribution of $\Upsilon$ in figure 11 .

The distributions at $R a=5 \cdot 10^{7}$ and $R a=10^{8}$ present two main modes (see figure $14-\mathrm{a}, \mathrm{b}, \mathrm{c}$ ). One mode is associated with the relatively low values described above. The other mode associated with a higher value corresponds to a long-lived switch from one metastable state to the other i.e a full reversal. There are more reversals and less cessations at $R a=10^{8}$ than at $R a=510^{7}$. A restriction of the histograms of the normalized time separation to the period $[0,1]$ is represented in figure $14-\mathrm{d}, \mathrm{e}, \mathrm{f})$ for the different Rayleigh numbers. It is of interest to compare these histograms, shown in Figure 11 which is extracted from application of periods of time, with the distributions of the indicator $\Upsilon$ strong similarities observed betwen the time histogram and the variations of $\Upsilon$ support the idea that the $\Upsilon$ indicator is able to discriminate between the true reversals of the flow (higher values) from shorter-lived transitions (lower values).

\section{Attractors and representative flow patterns}

We retain the local peaks method for reconstructing attractors. But taking advantage of previous knowledge about the physical response of the system [48, 56], we tune the peak identification process accordingly with physical considerations. First we extract the partial maxima from the time series of the absolute value of the angular momentum $|L|$. In order to avoid pointing a local peak during the transition period $\tau_{d}$ between two plateaus, we add a specific which differs with the Rayleigh number. The corresponding attractors are shown in Figure 15.

As expected, most of the points corresponding to the $\left(M_{i-1}, M_{i}\right)$ pairs are located close to the main diagonal for large $|L|$ values. This is particularly obvious at $R a=3 \cdot 10^{7}$ where the $L$ plateaus (i.e. fixed points) are very extended 
in time. Additional points show the path (limit cycle) between the opposite plateaus and point out transitional states quadrants of opposite signs. Fewer points are plotted in the center of the figure. They seems approximately equally distributed on the four quadrants for smaller $|L|$ values. For the two highest Rayleigh numbers attractors display more complex patterns. Transition path is still present. But points appears clustering around specific locations along the transition path as well as at the diagram center i.e. for low values of $L$.

Figure 16 shows a rough attempt of clustering the $\left(M_{i-1}, M_{i}\right)$ pairs for $R a=5 \cdot 10^{7}$, as well as the representative flow patterns of the $M_{i-1}$ and $M_{i}$ states, estimated from a conditional averaging over each cluster. Ten clusters (which can be related two by two by centrosymmetry) have been identified in the $\left(M_{i-1}, M_{i}\right)$ diagram: two fixed points at $|L| \simeq|\bar{L}|+\sigma(|L|)(|\bar{L}|$ and $\sigma(|L|)$ being the mean $|L|$ value and its standard deviation over the whole time series), two marginal fixed points at $|L| \lesssim|\bar{L}|-\sigma(|L|)$, and six clusters describing particular steps during the cycle limit.

As previously mentioned, the most dense clusters (in blue on the figure 16) which are located close to the main diagonal, are closely related to the two main fixed points i.e. plateaus. The evolution of the flow pattern between $M_{i-1}$ and $M_{i}$ states exhibits the growth of the corner flows. This physical phenomenon has been already identified in the literature (for example see [36, 48]) and associated to the accumulation phase of the generic reversal cycle in [56. This is clearly shown on figure 17 where examples of $\left(M_{i-1}, M_{i}\right)$ pairs are marked for each cluster on a particular sequence of the angular momentum time series at $R a=5 \cdot 10^{7}$. The orange cluster highlights the first part of the transition period (called the release phase in [56]), as the $M_{i}$ states point out the rebound instant of the transition on figure 17. On the contrary red clusters encompass the whole release phase from the reversal phenomenon to the following step consisting of the flow pattern reorganisation into the main diagonal roll surrounding by two smaller corner flows. The next cluster (in green) corresponds to the acceleration phase of the reversal cycle, where the large diagonal roll is being strengthened. Finally central clusters (in magenta) reveal the relative importance of the cessation regime in comparison to the plateau regime in $L$ time series. This regime is characterized by the disappearance of the main diagonal roll[48]. Figure 17 shows that the magenta points match well with rapid oscillations of $|L|$ peaks close to zero which is typical of cessations.

The clustering process has been applied similarly to both other attractors (figures 18, 19). It is noteworthy that the cluster shapes are quite similar whatever the Rayleigh number, as well as the corresponding representative flow patterns. However it can be noted that most clusters at $R a=3 \cdot 10^{7}$ does not contain a sufficient number of points to obtain a clear convergence of the representative flow patterns. Nonetheless the relative density of the clusters illustrates the relative time duration of different phases (as seen in figure 20). It shows shorter plateaus regarding the transition duration $\tau_{d}$ (stability decrease of the two main fixed points) as $R a$ increases, or the infrequency of cessations at $R a=3 \cdot 10^{7}$ and $10^{8}$. At all $R a$, the $M_{i-1}$ representative flow patterns of the clusters related to the release phase (in orange or red) point out a specific flow pattern where the corner flows occupy at least half the cavity width along the top or bottom wall. This feature is typical of the precursor event as noted in [55, 56. Consequently this methodology of snapshots classification appears to be able to discriminate the different regimes of reversal or cessation [48], the successive phases of standard reversals and specific events (rebound, precursor) [55, 56] as previously identified by using different techniques.

\section{DISCUSSION}

In this work embedding techniques and comparison with POD analysis have been carried out for the simulation of turbulent Rayleigh-Bénard convection in a 2D square cell for $\operatorname{Pr}=4.3$. Three different Rayleigh numbers were considered. Results suggest that significant information on the structure of the stationary states and their stability can be recovered by the embedding technique, which can be applied on-line to the time series of a single observable. The results are consistent with the characteristics of reversals provided by POD analysis.

The embedding theory displays a two-dimensional attractor, which is consistent with the idea that the reversals are independent from each other, at least at long-term as suggested by [52]. The existence of a two-dimensional description of the effective turbulent dynamics of the Rayleigh-Bénard flow is the first main result of this paper. In fact, contrary to Landau's conjecture [35], low dimensional descriptions of turbulent flow exist, providing that the right observable is embedded. This result also reinforces those found in [33] for the von Karman turbulent flow and in [58] for the turbulent atmospheric boundary layer. Moreover, the position on the reconstructed attractor can be used as a classifier of the different phases of the dynamics. The second main achievement of this paper is to show that the embedding/dynamical systems approach is still of interest for studying flow with a complex behavior. The novelty is to perform the embedding using the time series of a global observable tracing the symmetry of the flow, such as the angular momentum in the Rayleigh Bénard convection in the present case or the reduced frequency of rotation in the Von Karman flow [33]. Once the observable is identified it is a matter of seconds to embed the data and only a few 
minutes of CPU time on a laptop is needed to compute the stability $\Upsilon$. Finally, our study also suggests that in some phenomena, as those involving the motions of geophysical flows such as the atmosphere or the ocean, it can be difficult to directly identify a single symmetry parameter and in this case POD analysis can be helpful to identify the relevant variables to which the embedding technique could be applied. The use of Empirical Orthogonal Functions, which are analogous to POD modes, is already a common practice in the climate community. When possible, combining Proper Orthogonal Decomposition and embedding theory will therefore provide a robust description of the system.

\section{ACKNOWLEDGEMENTS}

This work was performed using HPC resources from GENCI-IDRIS (Grant 2a0326). DF was supported by ERC grant No. 338965. We thank the anonymous referees for their helpful suggestions.

* davide.faranda@cea.fr

$\dagger$ London Mathematical Laboratory, 14 Buckingham Street, London WC2N 6DF, UK

[1] J-P Eckmann, "Roads to turbulence in dissipative dynamical systems," Reviews of Modern Physics 53, 643 (1981).

[2] Pierre Bergé, Yves Pomeau, and Christian Vidal, Order within chaos (Wiley and Sons NY, 1984).

[3] Ian Mason, "A model for assessment of weather forecasts," Aust. Meteor. Mag 30, 291-303 (1982).

[4] Roberto Benzi, Giorgio Parisi, Alfonso Sutera, and Angelo Vulpiani, "Stochastic resonance in climatic change," Tellus 34, 10-15 (1982).

[5] Floris Takens, "Detecting strange attractors in turbulence," in Dynamical systems and turbulence, Warwick 1980 (Springer, 1981) pp. 366-381.

[6] Lyle Noakes, "The takens embedding theorem," International Journal of Bifurcation and Chaos 1, 867-872 (1991).

[7] Klaus Fraedrich, "Estimating the dimensions of weather and climate attractors," Journal of the atmospheric sciences 43 , 419-432 (1986).

[8] Edward N Lorenz, "Dimension of weather and climate attractors," Nature 353, 241 (1991).

[9] AA Tsonis, JB Elsner, and KP Georgakakos, "Estimating the dimension of weather and climate attractors: important issues about the procedure and interpretation," Journal of the atmospheric sciences 50, 2549-2555 (1993).

[10] Philip Holmes, John L Lumley, and Gal Berkooz, Turbulence, coherent structures, dynamical systems and symmetry (Cambridge university press, 1998).

[11] Franco Molteni, Roberto Buizza, Tim N Palmer, and Thomas Petroliagis, "The ecmwf ensemble prediction system: Methodology and validation," Quarterly journal of the royal meteorological society 122, 73-119 (1996).

[12] F Daviaud, J Hegseth, and P Bergé, "Subcritical transition to turbulence in plane couette flow," Physical review letters 69, $2511(1992)$.

[13] KD Hinsch, "Particle image velocimetry," Optical Engineering- New York-Marcel Dekker Incorporated 38, 235-235 (1993).

[14] Juan G Santiago, Steve T Wereley, Carl D Meinhart, DJ Beebe, and Ronald J Adrian, "A particle image velocimetry system for microfluidics," Experiments in fluids 25, 316-319 (1998).

[15] MJP Cullen, "The unified forecast/climate model," Meteorological Magazine 122, 81-94 (1993).

[16] Matti Hamalainen, Riitta Hari, Risto J Ilmoniemi, Jukka Knuutila, and Olli V Lounasmaa, "Magnetoencephalographytheory, instrumentation and applications to noninvasive studies of the working human brain," Reviews of modern Physics 65, 413 (1993).

[17] JM Haile, Molecular dynamics simulation, Vol. 18 (Wiley, New York, 1992).

[18] Peter J Steinbach and Bernard R Brooks, "Protein hydration elucidated by molecular dynamics simulation," Proceedings of the National Academy of Sciences 90, 9135-9139 (1993).

[19] Karl Pearson, "Principal components analysis," The London, Edinburgh and Dublin Philosophical Magazine and Journal 6, 566 (1901).

[20] Harold Hotelling, "Analysis of a complex of statistical variables into principal components." Journal of educational psychology 24, 417 (1933).

[21] DD Kosambi, "Statistics in function space," in DD Kosambi (Springer, 2016) pp. 115-123.

[22] Bérengère Podvin and John Lumley, "A low-dimensional approach for the minimal flow unit," Journal of Fluid Mechanics 362, 121-155 (1998).

[23] Edward N Lorenz, "Empirical orthogonal functions and statistical weather prediction," (1956).

[24] Lawrence Sirovich, "Turbulence and the dynamics of coherent structures. i. coherent structures," Quarterly of applied mathematics 45, 561-571 (1987).

[25] Gene H Golub and Charles F Van Loan, "Matrix computations, the john's hopkins univ," Press, Baltimore (1983).

[26] Harry H Harman, "Modern factor analysis." (1960).

[27] Bernard R Brooks, Dušanka Janežič, and Martin Karplus, "Harmonic analysis of large systems. i. methodology," Journal of computational chemistry 16, 1522-1542 (1995). 
[28] Norden E Huang, Zheng Shen, Steven R Long, Manli C Wu, Hsing H Shih, Quanan Zheng, Nai-Chyuan Yen, Chi Chao Tung, and Henry H Liu, "The empirical mode decomposition and the hilbert spectrum for nonlinear and non-stationary time series analysis," in Proceedings of the Royal Society of London A: Mathematical, Physical and Engineering Sciences, Vol. 454 (The Royal Society, 1998) pp. 903-995.

[29] RJ Bagley and Leon Glass, "Counting and classifying attractors in high dimensional dynamical systems," Journal of Theoretical Biology 183, 269-284 (1996).

[30] Roger Temam, Infinite-dimensional dynamical systems in mechanics and physics, Vol. 68 (Springer Science \& Business Media, 2012).

[31] Ludwig Arnold, Random dynamical systems (Springer Science \& Business Media, 2013).

[32] Mickaël D Chekroun, Honghu Liu, and Shouhong Wang, Approximation of Stochastic Invariant Manifolds: Stochastic Manifolds for Nonlinear SPDEs I. (Springer, 2015).

[33] Davide Faranda, Yuzuru Sato, Brice Saint-Michel, Cecile Wiertel, Vincent Padilla, Bérengère Dubrulle, and François Daviaud, "Stochastic chaos in a turbulent swirling flow," Physical review letters 119, 014502 (2017).

[34] Edward N Lorenz, "Deterministic nonperiodic flow," Journal of the atmospheric sciences 20, $130-141$ (1963).

[35] Lev D Landau, "On the problem of turbulence," in Dokl. Akad. Nauk SSSR, Vol. 44 (1944) pp. $339-349$.

[36] Kazuyasu Sugiyama, Rui Ni, Richard JAM Stevens, Tak Shing Chan, Sheng-Qi Zhou, Heng-Dong Xi, Chao Sun, Siegfried Grossmann, Ke-Qing Xia, and Detlef Lohse, "Flow reversals in thermally driven turbulence," Physical review letters 105, 034503 (2010).

[37] David Ruelle and Floris Takens, "On the nature of turbulence," Communications in mathematical physics 20, 167-192 (1971).

[38] Liangyue Cao, "Practical method for determining the minimum embedding dimension of a scalar time series," Physica D: Nonlinear Phenomena 110, 43-50 (1997).

[39] Norman H Packard, James P Crutchfield, J Doyne Farmer, and Robert S Shaw, "Geometry from a time series," Physical review letters 45, 712 (1980).

[40] Holger Kantz and Thomas Schreiber, Nonlinear time series analysis, Vol. 7 (Cambridge university press, 2004).

[41] Davide Faranda, Flavio Maria Emanuele Pons, Eugenio Giachino, Sandro Vaienti, and Bérengère Dubrulle, "Early warnings indicators of financial crises via auto regressive moving average models," Communications in Nonlinear Science and Numerical Simulation 29, 233-239 (2015).

[42] Ser-Huang Poon and Clive WJ Granger, "Forecasting volatility in financial markets: A review," Journal of economic literature 41, 478-539 (2003).

[43] Hirotugu Akaike, "Time series analysis and control through parametric models," Applied Time Series Analysis , 1-23 (1978).

[44] Hirotogu Akaike, "Information theory and an extension of the maximum likelihood principle," in Selected Papers of Hirotugu Akaike (Springer, 1998) pp. 199-213.

[45] D. Faranda and D. Defrance, "A wavelet-based approach to detect climate change on the coherent and turbulent component of the atmospheric circulation," Earth System Dynamics 7, 517-523 (2016).

[46] P. Holmes, J.L. Lumley, Gal Berkooz, and C. W. Rowley, Turbulence, Coherent Structures, Dynamical Systems and Symmetry (Cambridge University Press, 2012).

[47] S. Xin, J. Chergui, and P. Le Quéré, "3d spectral parallel multi-domain computing for natural convection flows," in Parallel Computational Fluid Dynamics 2008 (2010).

[48] Bérengère Podvin and Anne Sergent, "A large-scale investigation of wind reversal in a square rayleigh-bénard cell," Journal of Fluid Mechanics 766, 172-201 (2015).

[49] Mani Chandra and Mahendra K Verma, "Flow reversals in turbulent convection via vortex reconnections," Physical review letters 110, 114503 (2013).

[50] Rui Ni, Shi-Di Huang, and Ke-Qing Xia, "Reversals of the large-scale circulation in quasi-2d rayleigh-bénard convection," Journal of Fluid Mechanics 778, R5 (2015).

[51] Eric Brown, Alexei Nikolaenko, and Guenter Ahlers, "Reorientation of the large-scale circulation in turbulent rayleighbénard convection," Physical review letters 95, 084503 (2005).

[52] Katepalli R Sreenivasan, Alexander Bershadskii, and JJ Niemela, "Mean wind and its reversal in thermal convection," Physical Review E 65, 056306 (2002).

[53] Francisco Fontenele Araujo, Siegfried Grossmann, and Detlef Lohse, "Wind reversals in turbulent rayleigh-bénard convection," Physical review letters 95, 084502 (2005).

[54] Roberto Benzi, "Flow reversal in a simple dynamical model of turbulence," Physical review letters 95, 024502 (2005).

[55] Bérengère Podvin and Anne Sergent, "Precursor for wind reversal in a square Rayleigh-Bénard cell," Physical Review E 95, 013112 (2017).

[56] Andres Castillo-Castellanos, Anne Sergent, and Maurice Rossi, "Reversal cycle in square rayleigh-bénard cells in turbulent regime," Journal of Fluid Mechanics 808, 614-640 (2016).

[57] Heng-Dong Xi and Ke-Qing Xia, "Flow mode transitions in turbulent thermal convection," Physics of Fluids 20, 055104 (2008).

[58] Guillaume Nevo, Nikki Vercauteren, Amandine Kaiser, Bérengère Dubrulle, and Davide Faranda, "Statistical-mechanical approach to study the hydrodynamic stability of the stably stratified atmospheric boundary layer," Physical Review Fluids 2, 084603 (2017). 


\begin{tabular}{|c|c|c|c|c|c|}
\hline$R a$ & $\left(N_{x}, N_{z}\right)$ & $\Delta t$ & $N_{\text {snapshots }}$ & $\Delta T_{\text {snapshots }}$ & $N_{\text {total }}$ \\
\hline $3 \cdot 10^{7}$ & $(159,385)$ & $610^{-4}$ & 825 & 6 & 12000 \\
\hline $5 \cdot 10^{7}$ & $(159,385)$ & $610^{-4}$ & 825 & 6 & 12000 \\
\hline $10^{8}$ & $(201,513)$ & $310^{-4}$ & 660 & 4.5 & 8000 \\
\hline
\end{tabular}

TABLE I. Simulation and POD analysis characteristics ar different Rayleigh numbers : numerical resolution, time step, number of snapshots used to extract the POD eigenfunctions, time separation between snapshots, total number of snapshots considered in the time series

\begin{tabular}{|c|c|c|c|c|}
\hline$R a$ & $\lambda^{1}$ & $\lambda^{2}$ & $\lambda^{3}$ & $\lambda^{4}$ \\
\hline $3 \cdot 10^{7}$ & 0.7 & 0.13 & 0.02 & 0.01 \\
\hline $5 \cdot 10^{7}$ & 1.15 & 0.26 & 0.17 & 0.05 \\
\hline $10^{8}$ & 1.07 & 0.26 & 0.15 & 0.05 \\
\hline
\end{tabular}

TABLE II. POD first eigenvalues at different Rayleigh numbers.

\begin{tabular}{|c|c|c|c|}
\hline$R a$ & $\sum_{\lambda \geq 5} / \sum_{\lambda}$ & $\langle T\rangle$ & $d_{e q}^{r m s}$ \\
\hline $3 \cdot 10^{7}$ & 0.09 & 1160 & 0.09 \\
\hline $5 \cdot 10^{7}$ & 0.12 & 200 & 0.38 \\
\hline $10^{8}$ & 0.10 & 195 & 0.30 \\
\hline
\end{tabular}

TABLE III. POD Characteristics at different Rayleigh numbers. $\langle T\rangle$ is defined as the time-averaged value separating two zeros of $a^{1}$ and is expressed in convective time units. $d_{e q}^{r m s}$ is defined as the room mean square value of the minimal distance between the POD state vector $\left\{a^{i}, 1 \leq i \leq 4\right\}$ and one of the steady states. 
a)

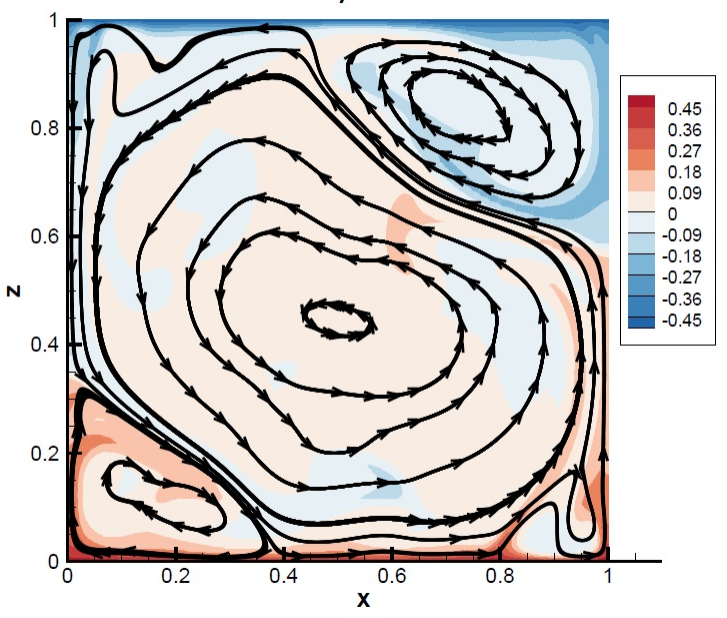

b)

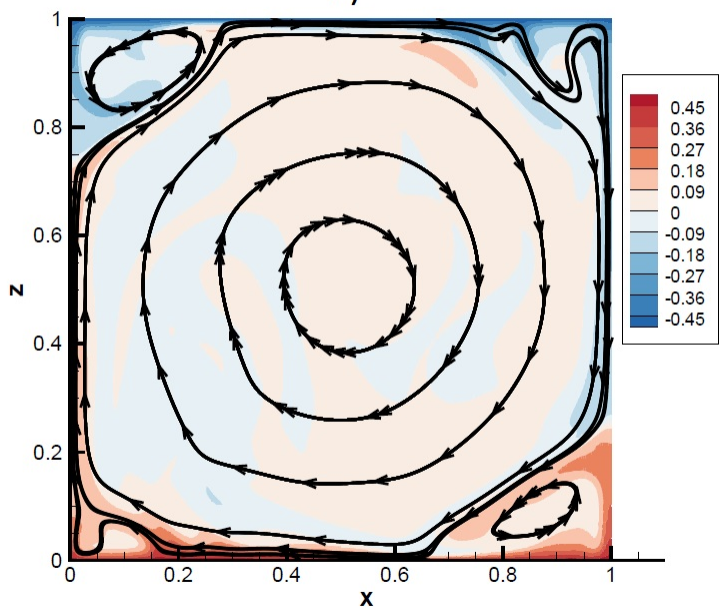

FIG. 1. Instantaneous flow field in the $\mathbf{2 D}$ Rayleigh-Bénard cell at $R a=5 \cdot 10^{7}$ for two arbitrary times preceding (a) and following (b) a reversal; Flow streamlines and temperature isocontours. 
a)

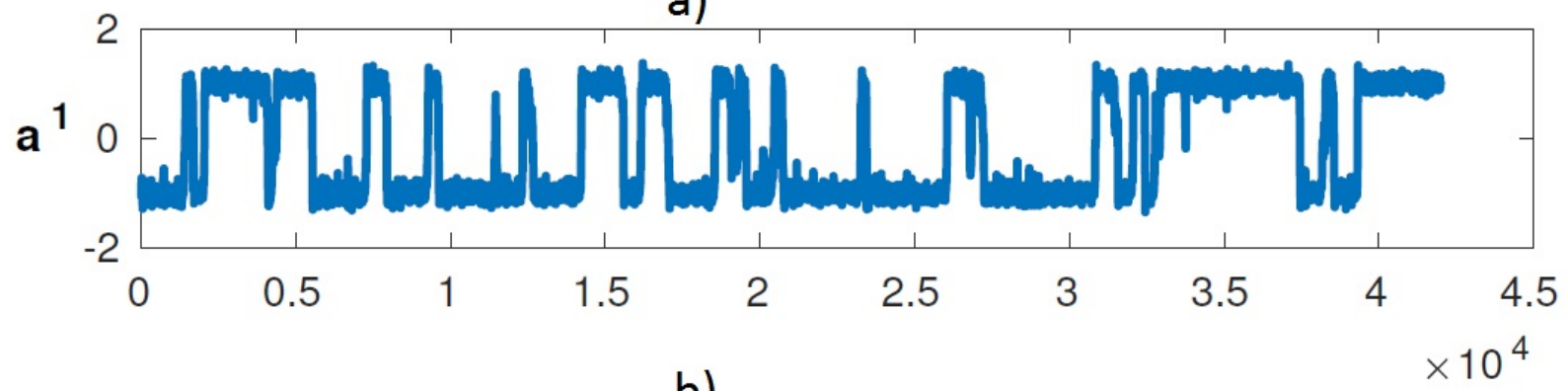

b)

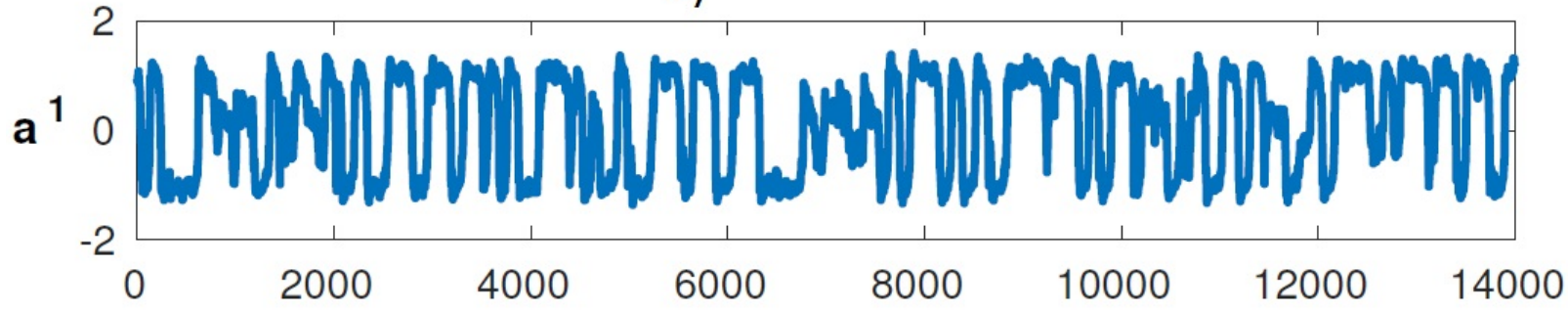

c)

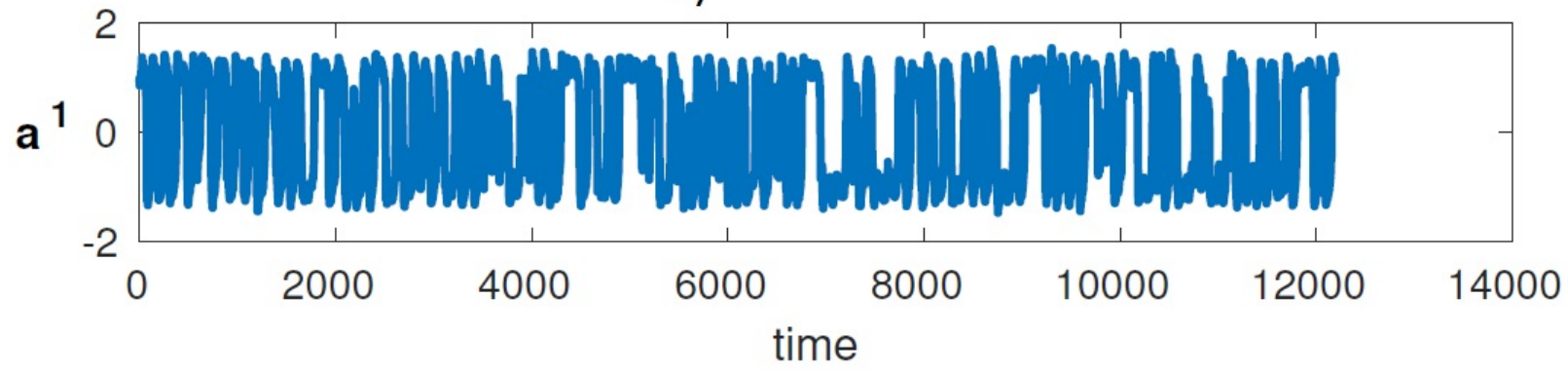

FIG. 2. Time evolution of first POD mode $a^{1}$. a) $R a=3 \cdot 10^{7}$, b) $R a=5 \cdot 10^{7}$, c) $R a=10^{8}$.

a)

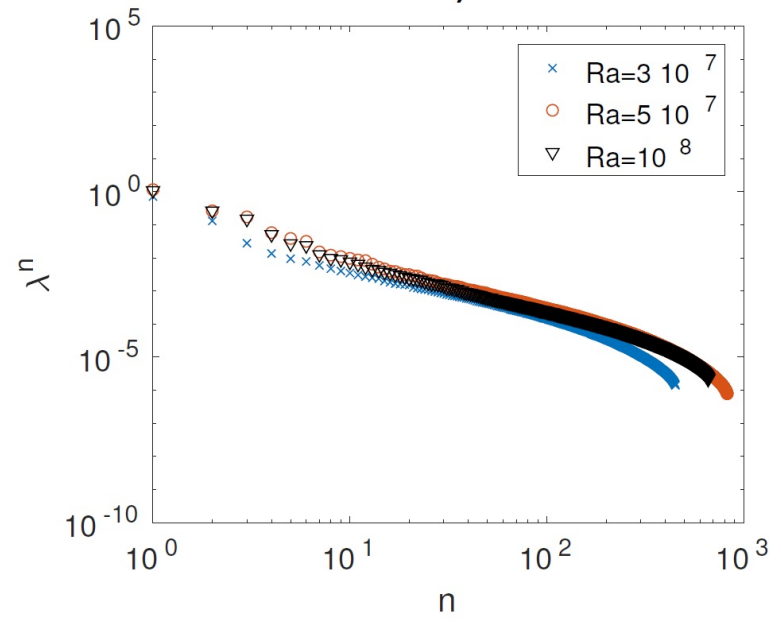

b)

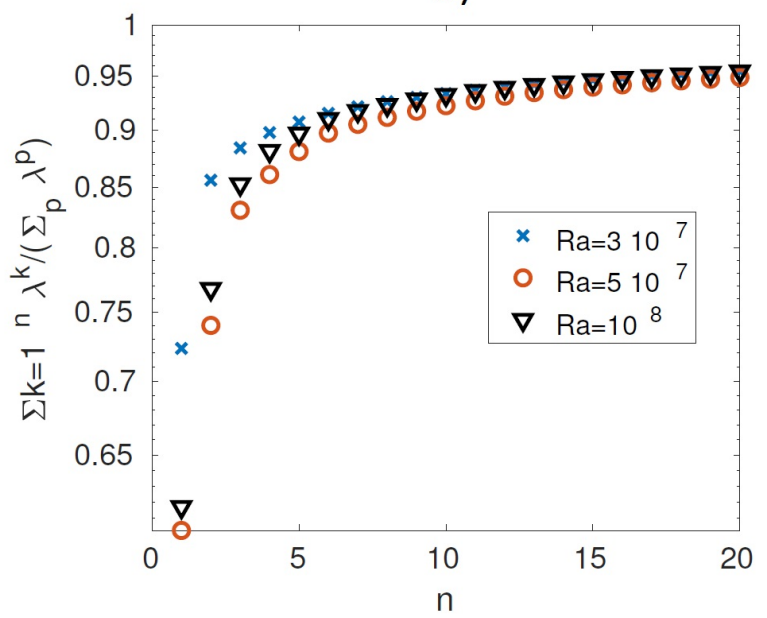

FIG. 3. a) POD spectrum at the different Rayleigh numbers - the eigenvalues are unnormalized. ; b) Relative fraction of POD energy contained in the first 20 modes. 

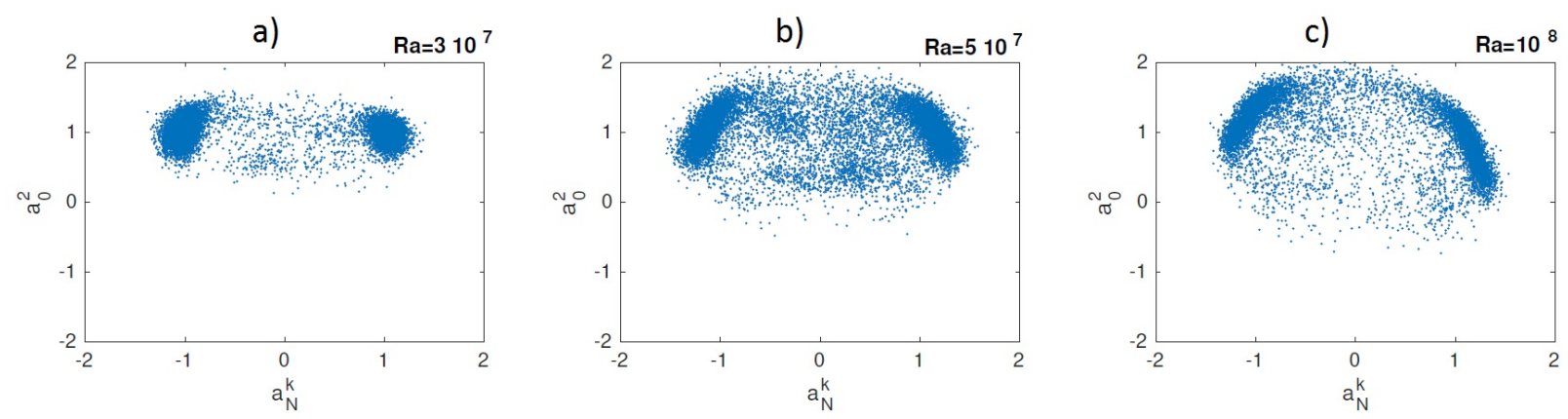

FIG. 4. Phase portraits for the first two normalized POD modes $a_{N}^{1}$ and $a_{N}^{2}$ (the $N$ subscript stands for normalization). a) $R a=3 \cdot 10^{7}$, b) $R a=5 \cdot 10^{7}$, c) $R a=10^{8}$.
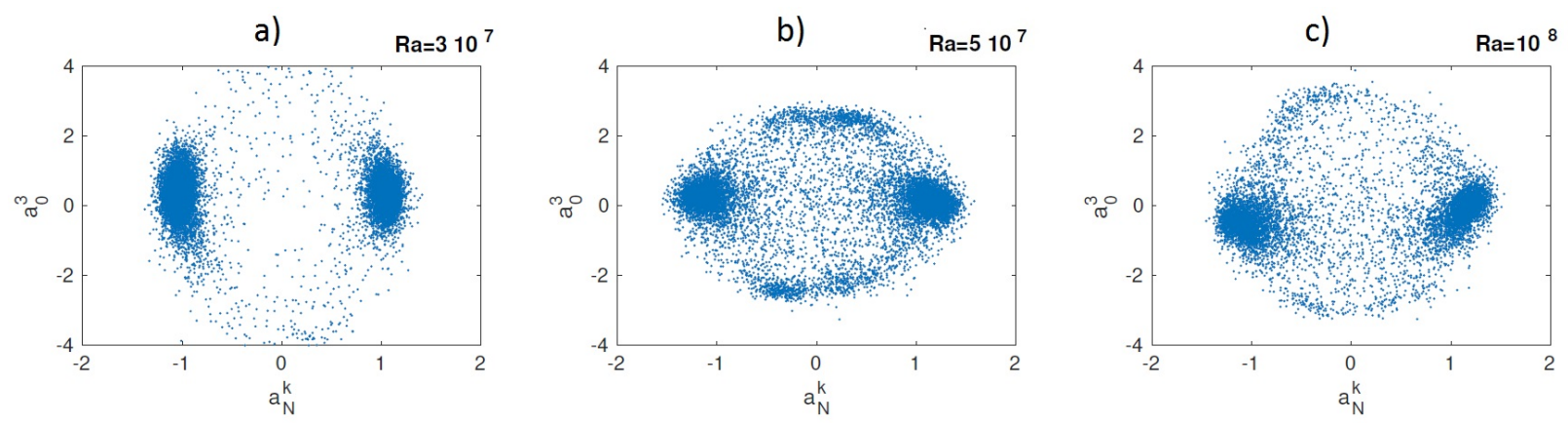

FIG. 5. Phase portraits for the first two normalized POD modes $a_{N}^{1}$ and $a_{N}^{3}$ (the $N$ subscript stands for normalization). a) $R a=3 \cdot 10^{7}$, b) $R a=5 \cdot 10^{7}$, c) $R a=10^{8}$.
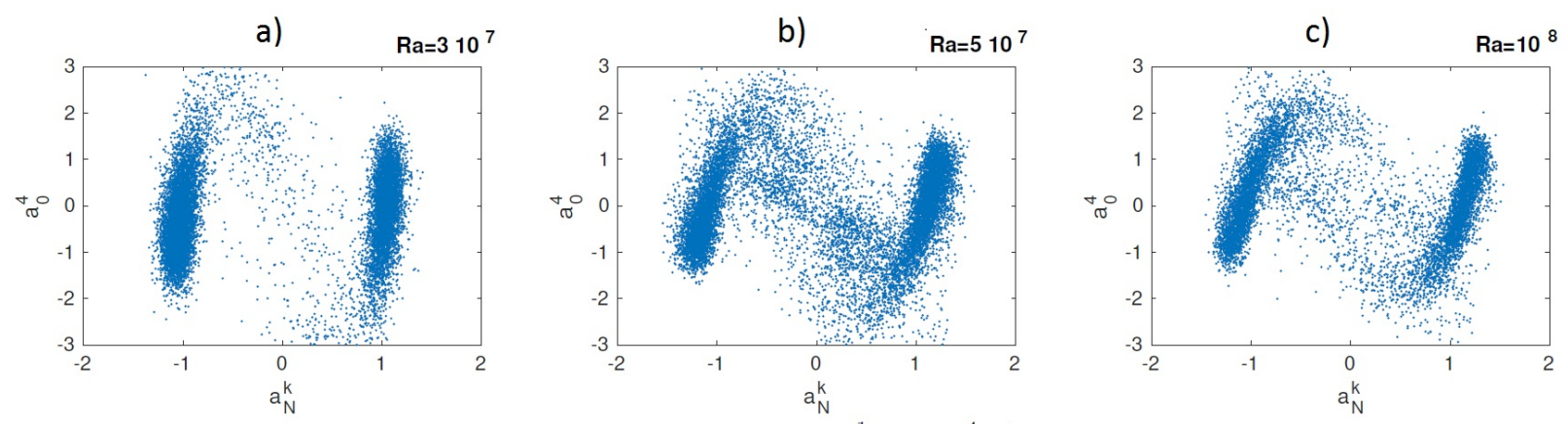

FIG. 6. Phase portraits for the first two normalized POD modes $a_{N}^{1}$ and $a_{N}^{4}$ (the $N$ subscript stands for normalization). a) $R a=3 \cdot 10^{7}$, b) $R a=5 \cdot 10^{7}$, c) $R a=10^{8}$. 
a)

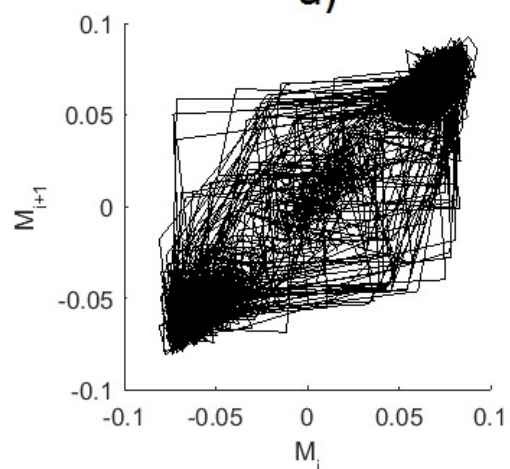

d)
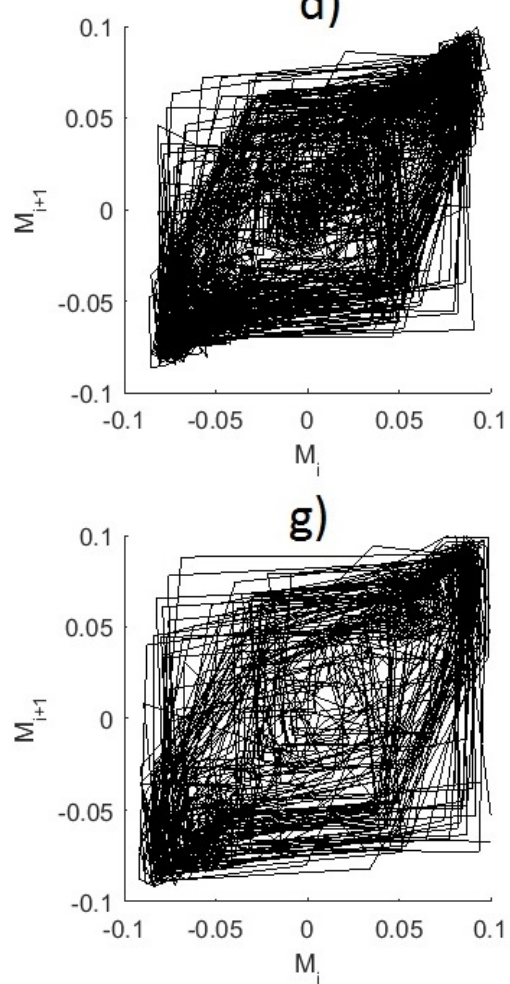

b)

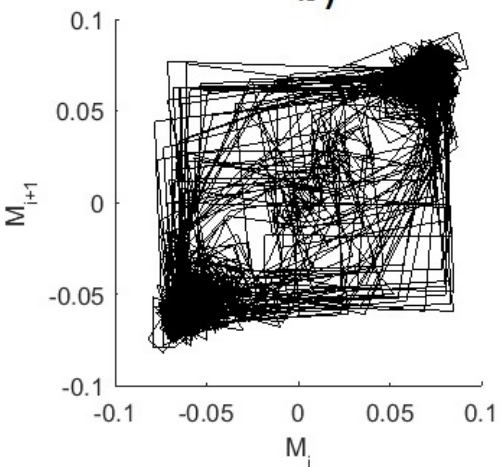

e)

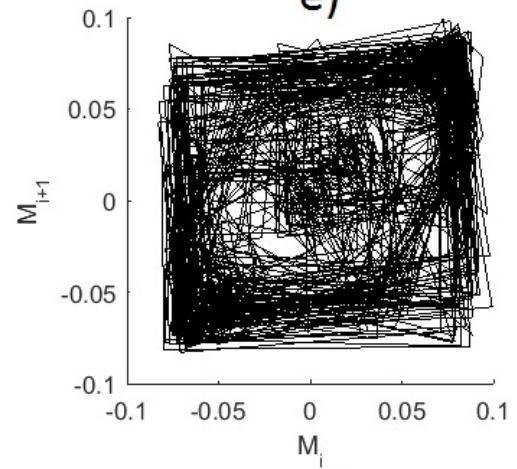

h)

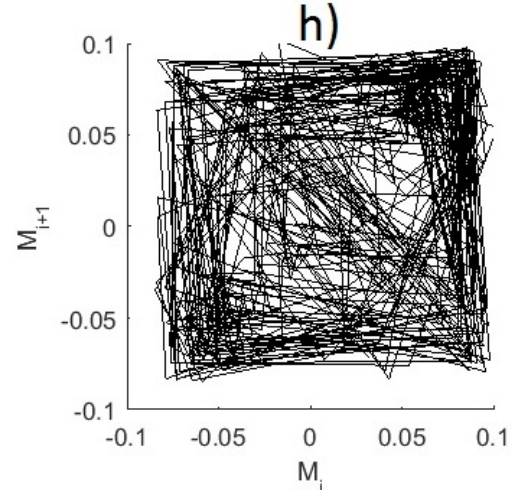

c)

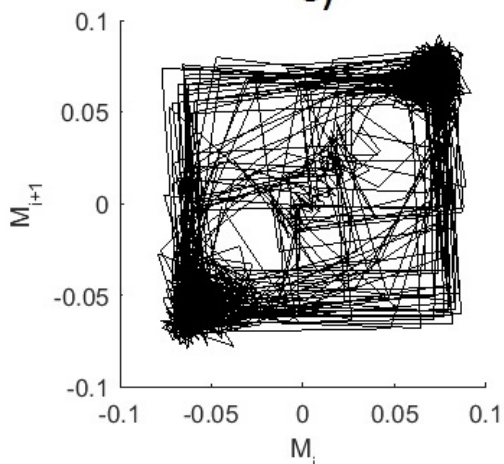

f)

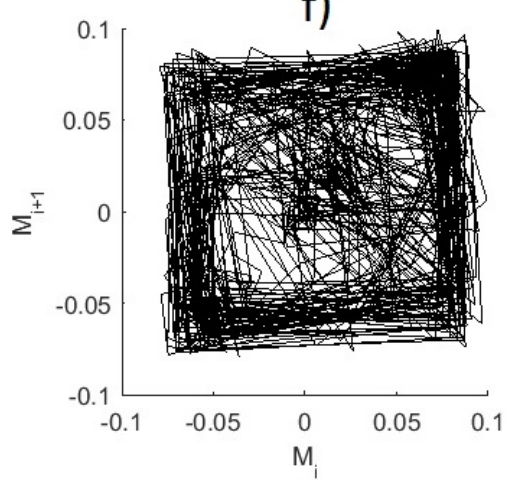

i)

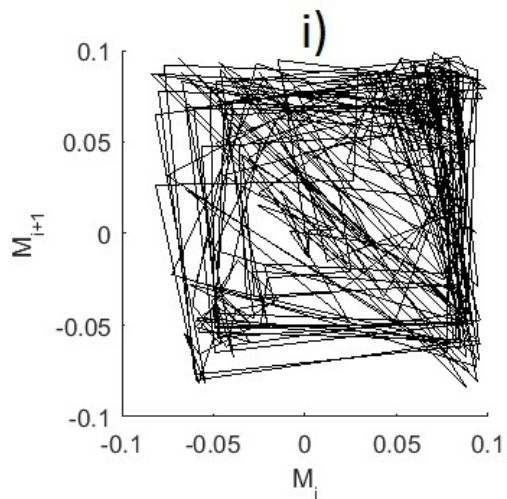

FIG. 7. Rayleigh-Bénard attractors obtained at $R a=3 \cdot 10^{7}$ (a,b,c), $R a=5 \cdot 10^{7}$ (d,e,f), $R a=1 \cdot 10^{8}$ (g,h,i) varying $\Delta t$ from $\Delta t=3(\mathrm{a}, \mathrm{d}, \mathrm{g}), \Delta t=6(\mathrm{~b}, \mathrm{e}, \mathrm{h})$ and $\Delta t=9(\mathrm{c}, \mathrm{f}, \mathrm{i})$. 




FIG. 8. Rayleigh-Bénard time series obtained at $R a=3 \cdot 10^{7}$ (a), $R a=5 \cdot 10^{7}$ (b), $R a=1 \cdot 10^{8}$ (c) for $\Delta t=3$. The colorscale represents the value of $\Upsilon$.

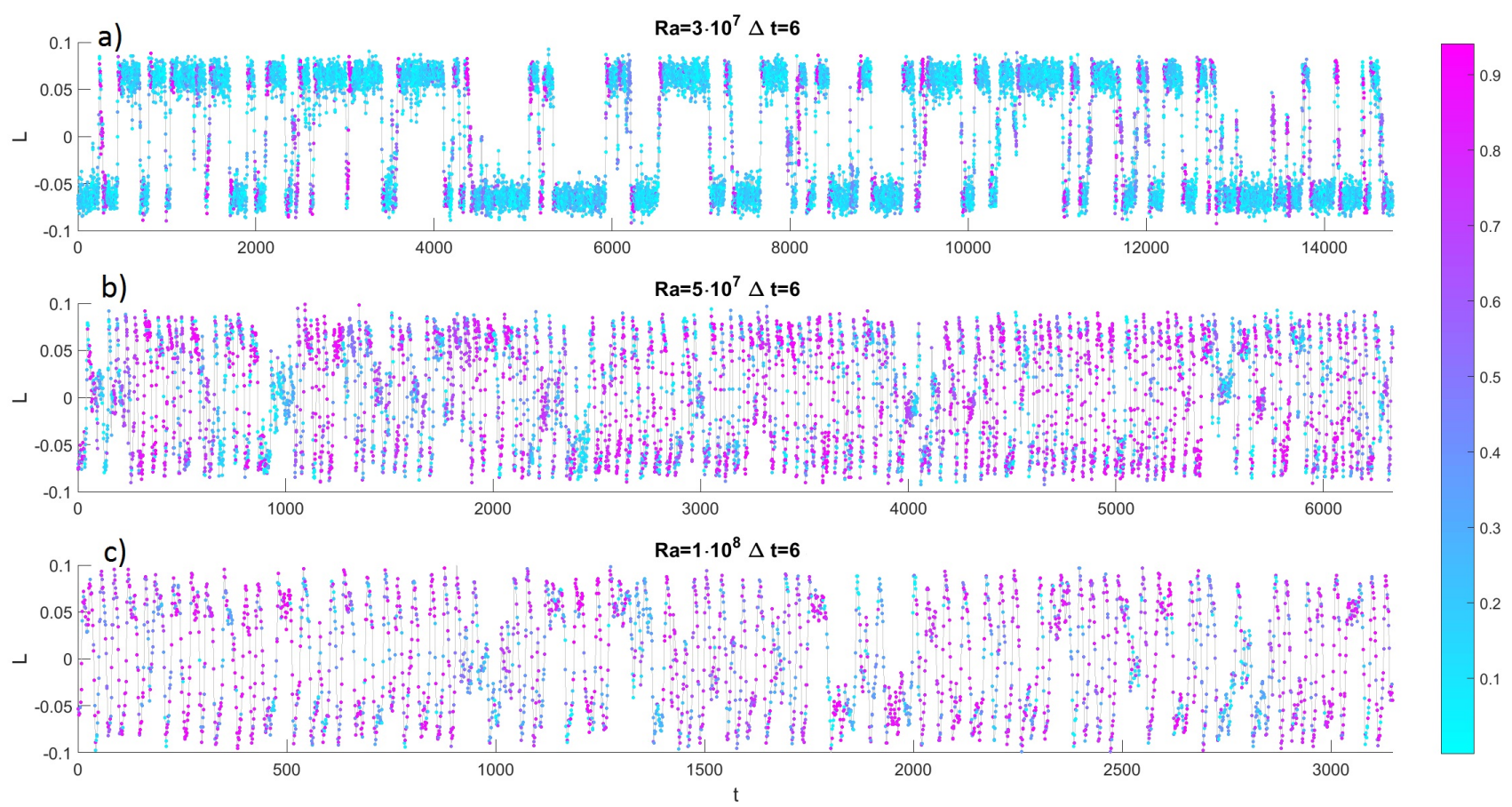

FIG. 9. Rayleigh-Bénard time series obtained at $R a=3 \cdot 10^{7}$ (a), $R a=5 \cdot 10^{7}$ (b), $R a=1 \cdot 10^{8}$ (c) for $\Delta t=6$. The colorscale represents the value of $\Upsilon$ 


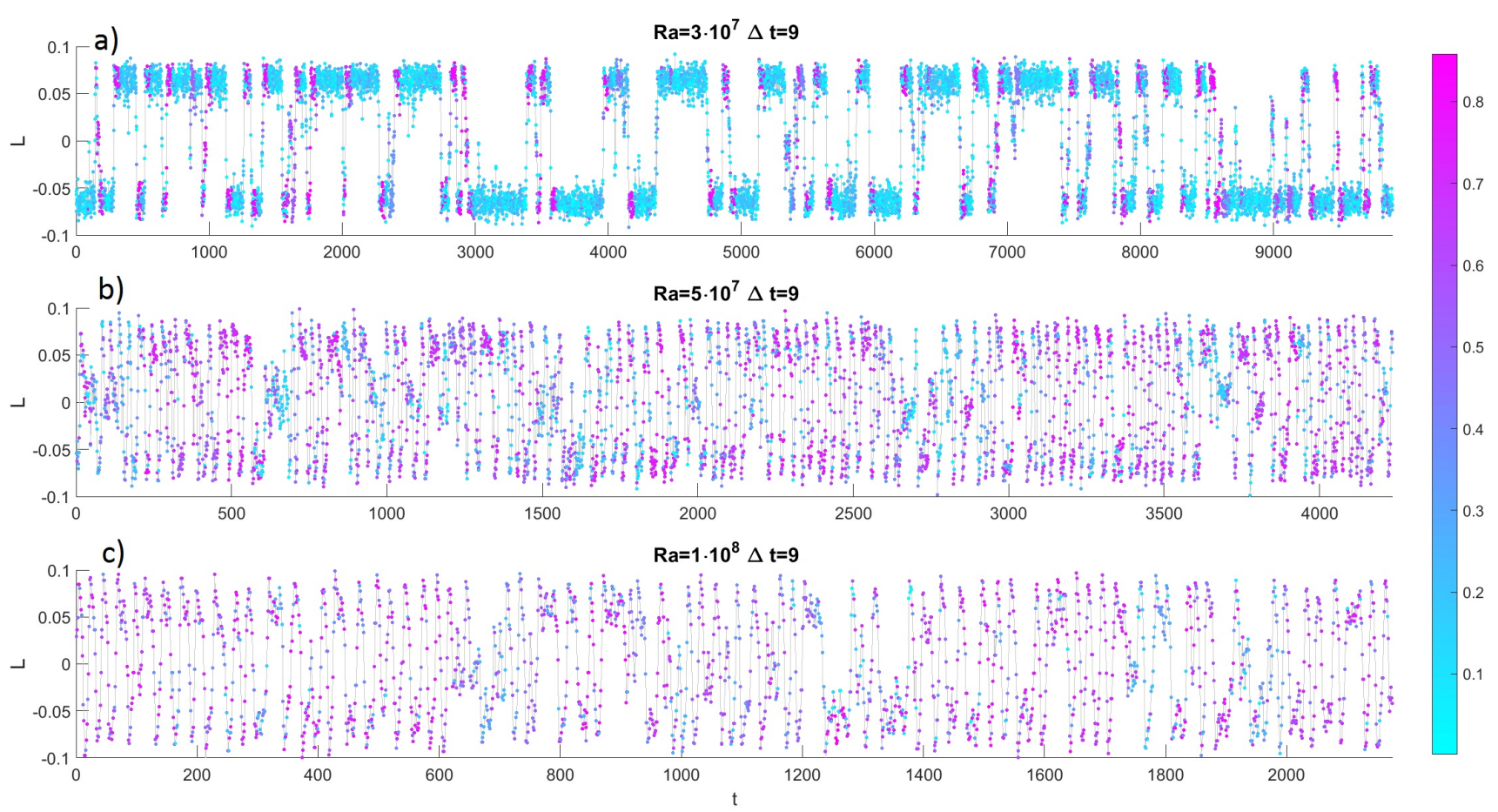

FIG. 10. Rayleigh-Bénard time series obtained at $R a=3 \cdot 10^{7}$ (a), $R a=5 \cdot 10^{7}$ (b), $R a=1 \cdot 10^{8}$ (c) for $\Delta t=9$. The colorscale represents the value of $\Upsilon$
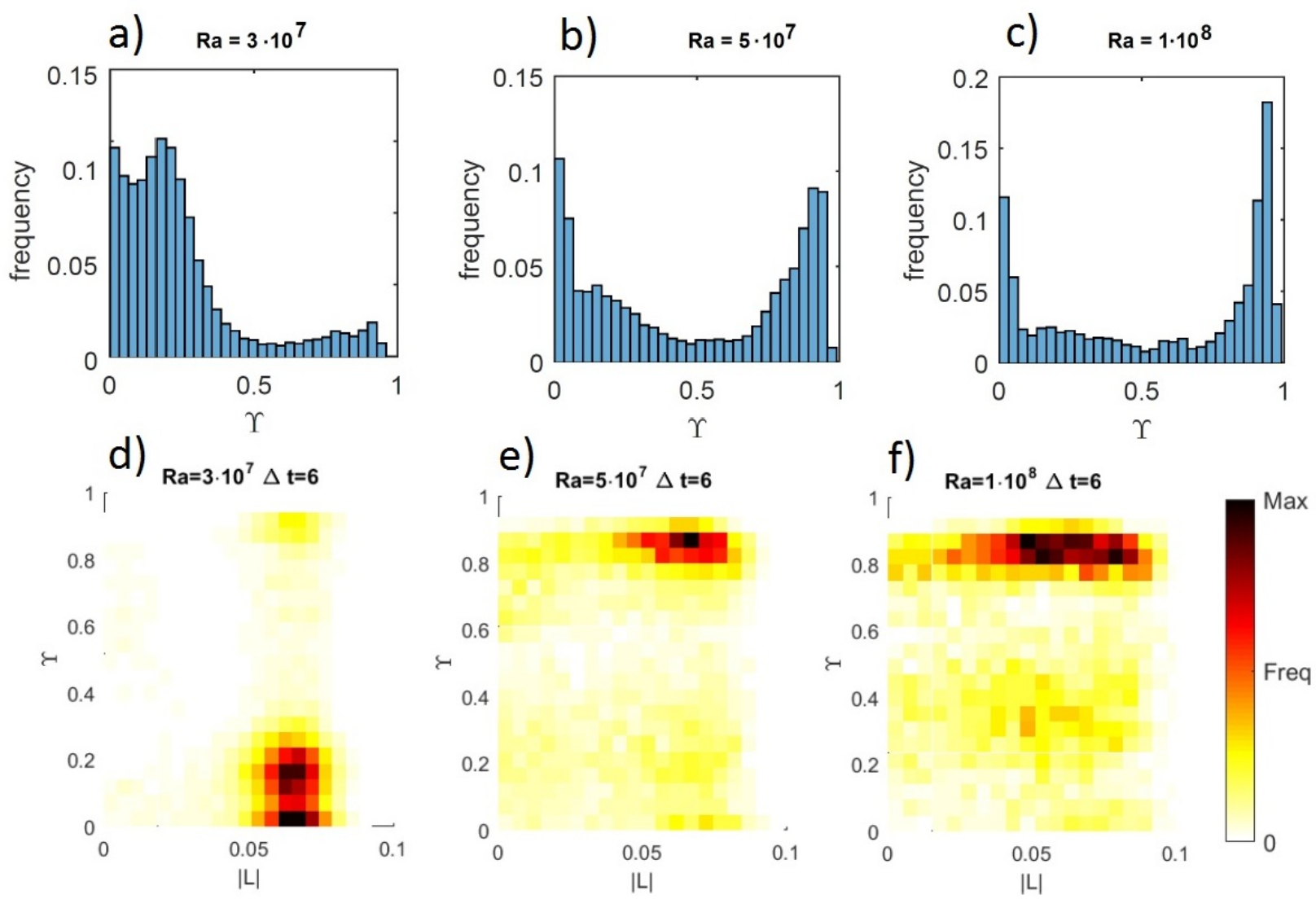

FIG. 11. a,b,c): histograms of $\Upsilon$. d,e,f): bivariate histograms $\Upsilon$ vs $L$. $R a=3 \cdot 10^{7}$ (a), $R a=5 \cdot 10^{7}$ (b), $R a=1 \cdot 10^{8}(\mathrm{c})$. 
a)
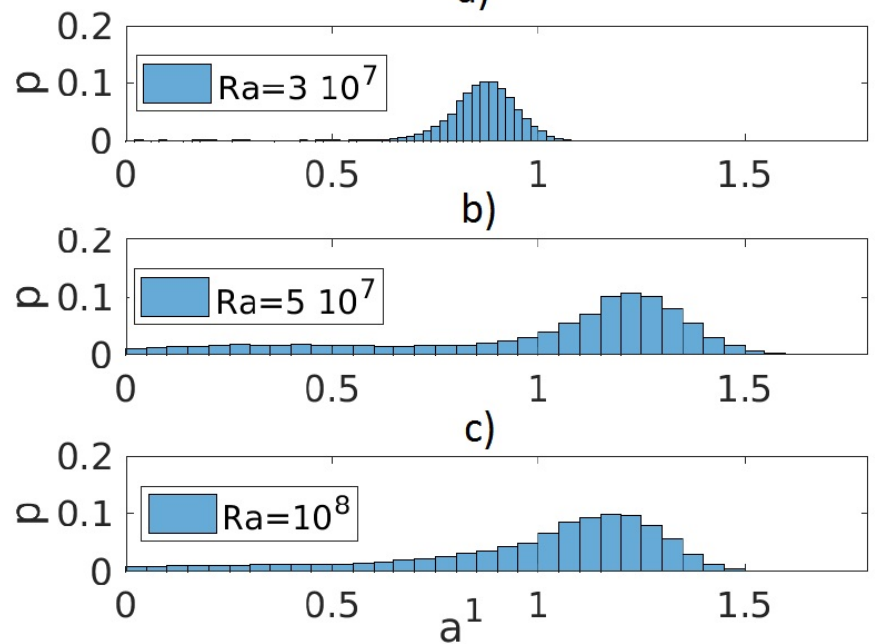

FIG. 12. Histogram of the POD mode $a^{1}$. a) $R a=3 \cdot 10^{7}$, b) $R a=5 \cdot 10^{7}$, c) $R a=10^{8}$.

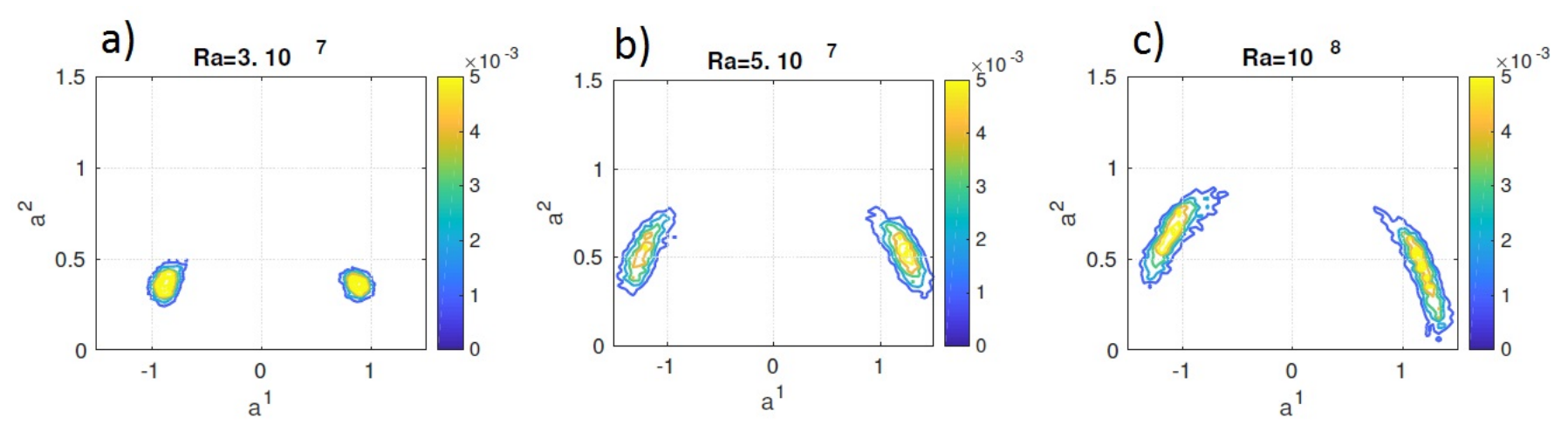

FIG. 13. Joint histogram of the two POD modes $a^{1}$ vs $a^{2}$. a) $R a=3 \cdot 10^{7}$, b) $R a=5 \cdot 10^{7}$, c) $R a=10^{8}$. 
a)

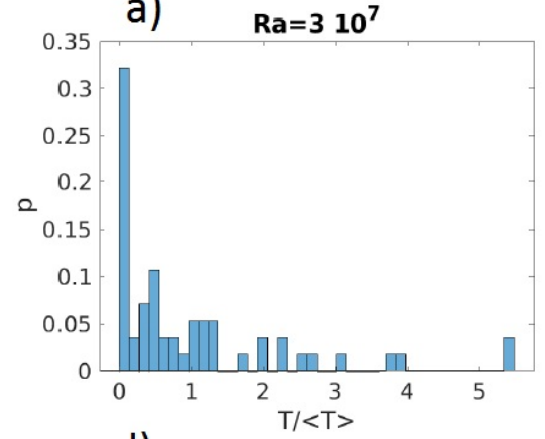

d) $\quad \operatorname{Ra}=310^{7}$

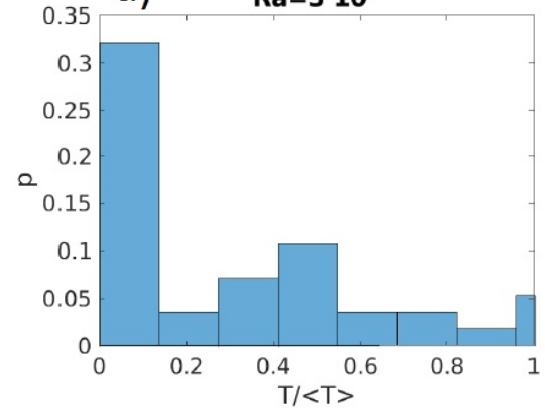

b)

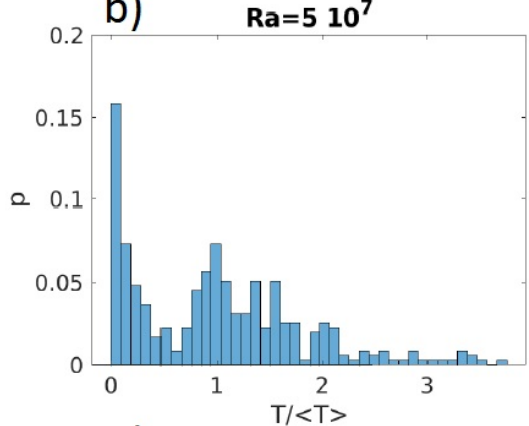

e) $\quad R a=510^{7}$

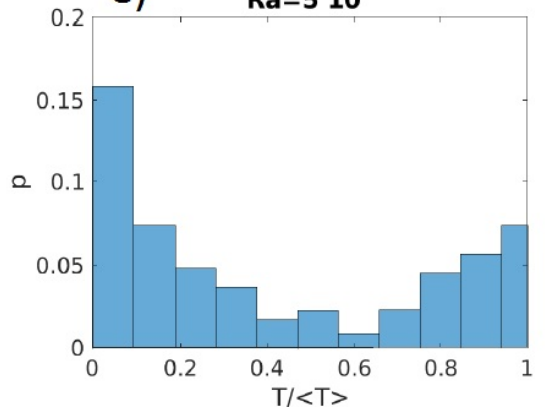

c)
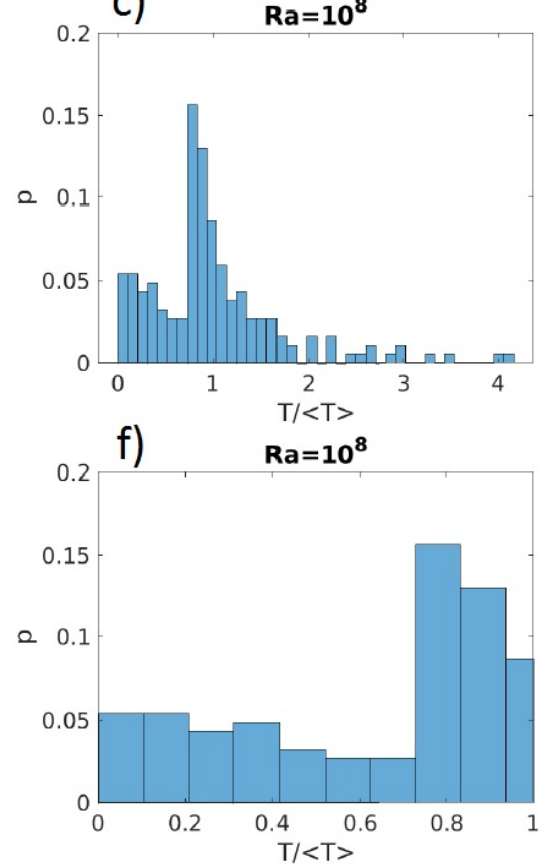

FIG. 14. a,b,c): Distribution of time (normalized by time-averaged value) between zeros of first POD mode $a^{1}$; d,e,f): Distribution of normalized time between zeros of first POD mode $a^{1}$ restricted to the time $[0, T /<T>]$.
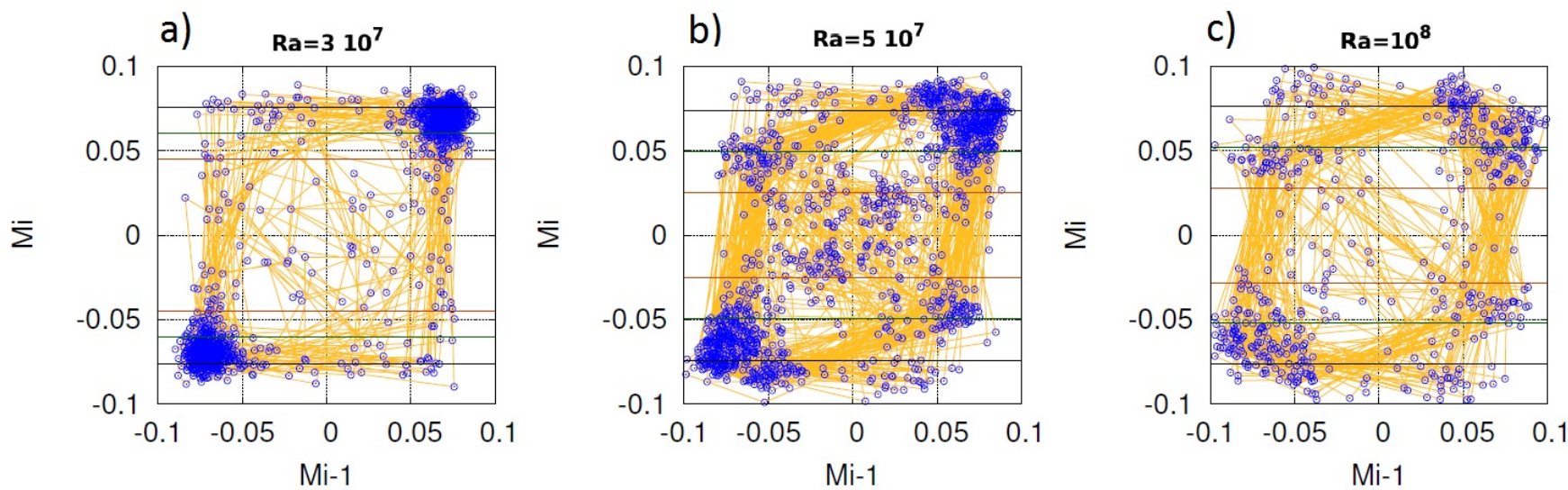

FIG. 15. Rayleigh-Bénard attractors obtained at $R a=3 \cdot 10^{7}$ (a), $R a=5 \cdot 10^{7}$ (b), $R a=10^{8}$ (c) with a time between subsequent peaks larger than twice the transition time $\tau_{d}\left(\tau_{d} \sim 30,12.5,15\right.$ convective time units for $R a=3 \cdot 10^{7}, 5 \cdot 10^{7}, 10^{8}$ respectively). Yellow lines show the paths between two subsequent $\left(M_{i-1}, M_{i}\right)$ points. 


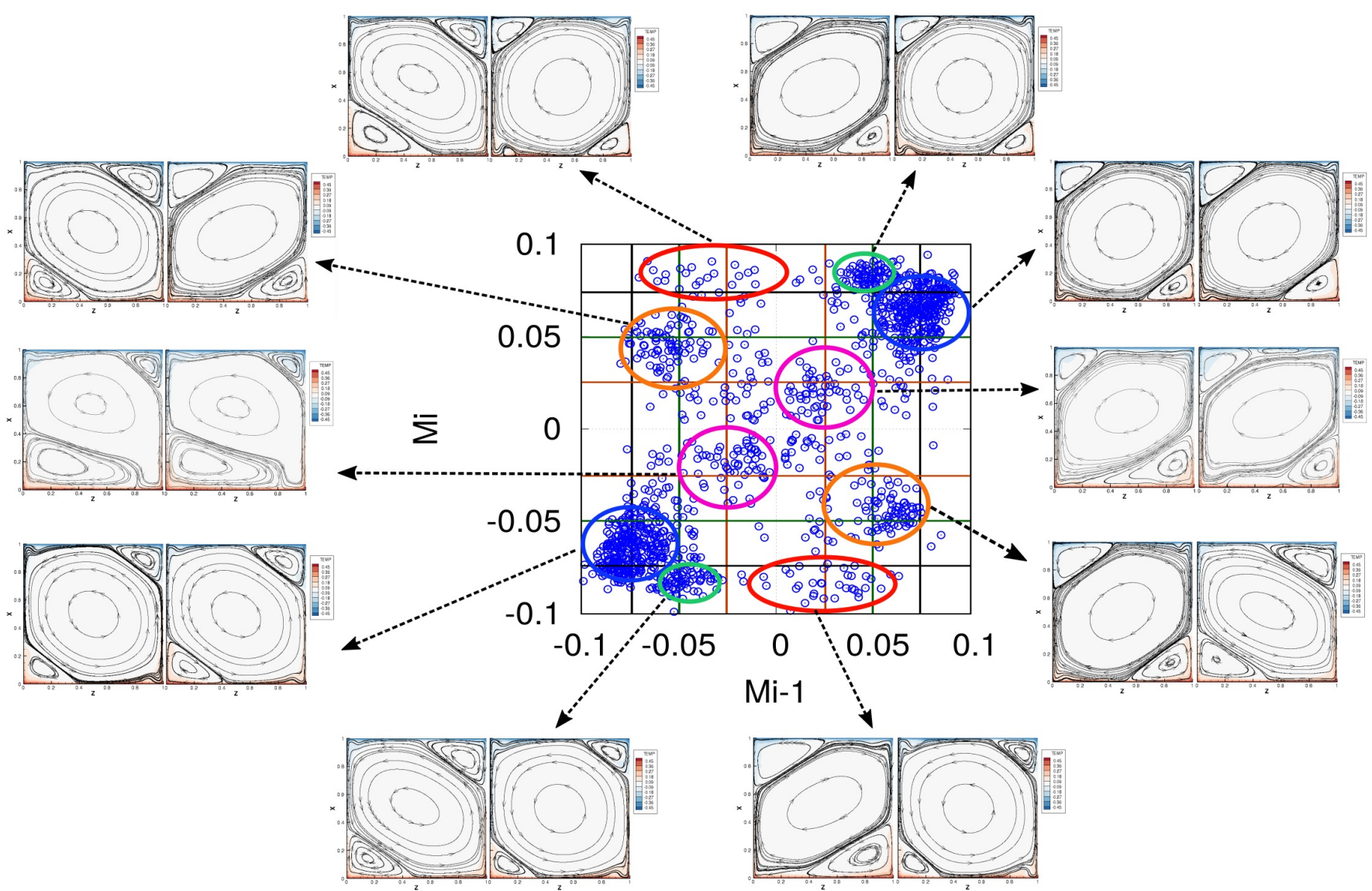

FIG. 16. Clusters in the Rayleigh-Bénard attractor at $R a=5 \cdot 10^{7}$ and the related mean flow patterns at $M_{i-1}$ (left) and $M_{i}$ (right). Colour of cluster refers to the different regime or phases of the reversal cycle : first part of the release (orange), complete release (red), acceleration (green), accumulation (blue) and cessation (magenta). Flow pattern is shown by streamlines and temperature isocontours. Green, orange and black straight solid lines correspond to $|\bar{L}|,|\bar{L}|-\sigma(|L|)$ and $|\bar{L}|+\sigma(|L|)$ respectively.

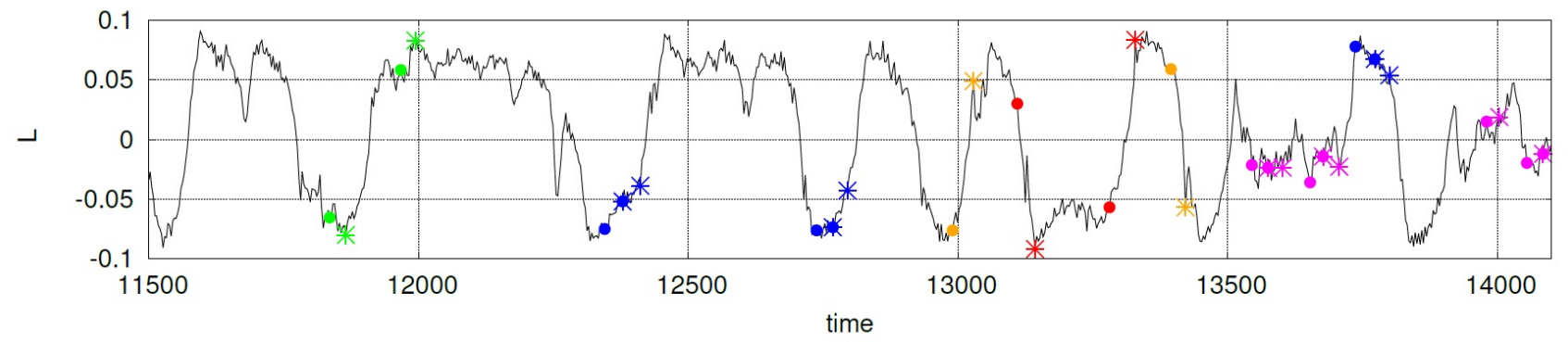

FIG. 17. Particular sequence of the angular momentum $L$ time series at $R a=5 \cdot 10^{7}$. Colours refer to the different regime or phases of the reversal cycle : release (orange), after rebound (red), acceleration (green), accumulation (blue) and cessation (magenta). Markers give examples of the pairs $\left(M_{i-1}, M_{i}\right)$ for each cluster: $\bullet\left(M_{i-1}\right), *(M i)$. 


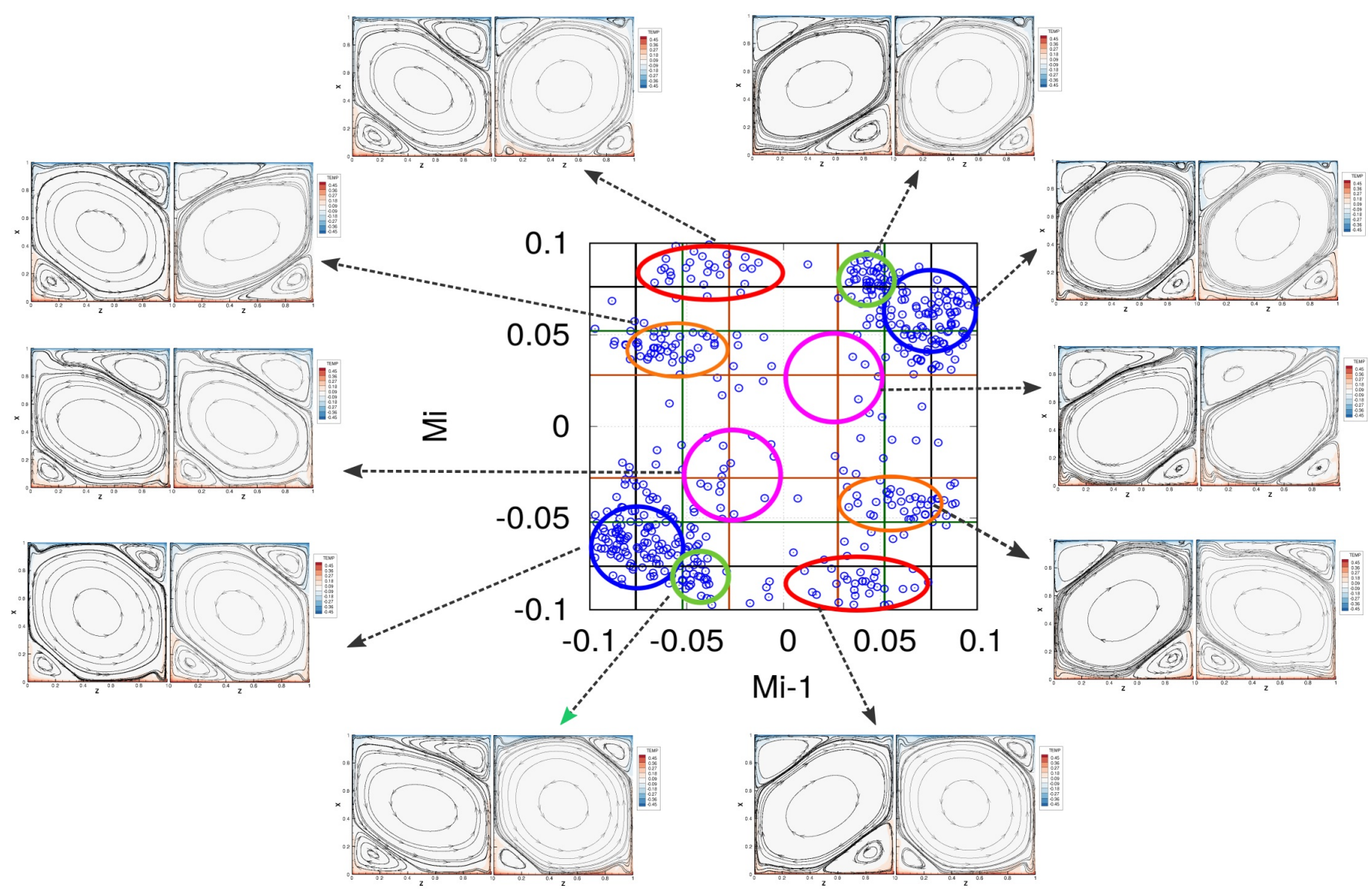

FIG. 18. Clusters in Rayleigh-Bénard attractors at $R a=10^{8}$ and the related mean flow patterns. Same legend as in figure 16 . 


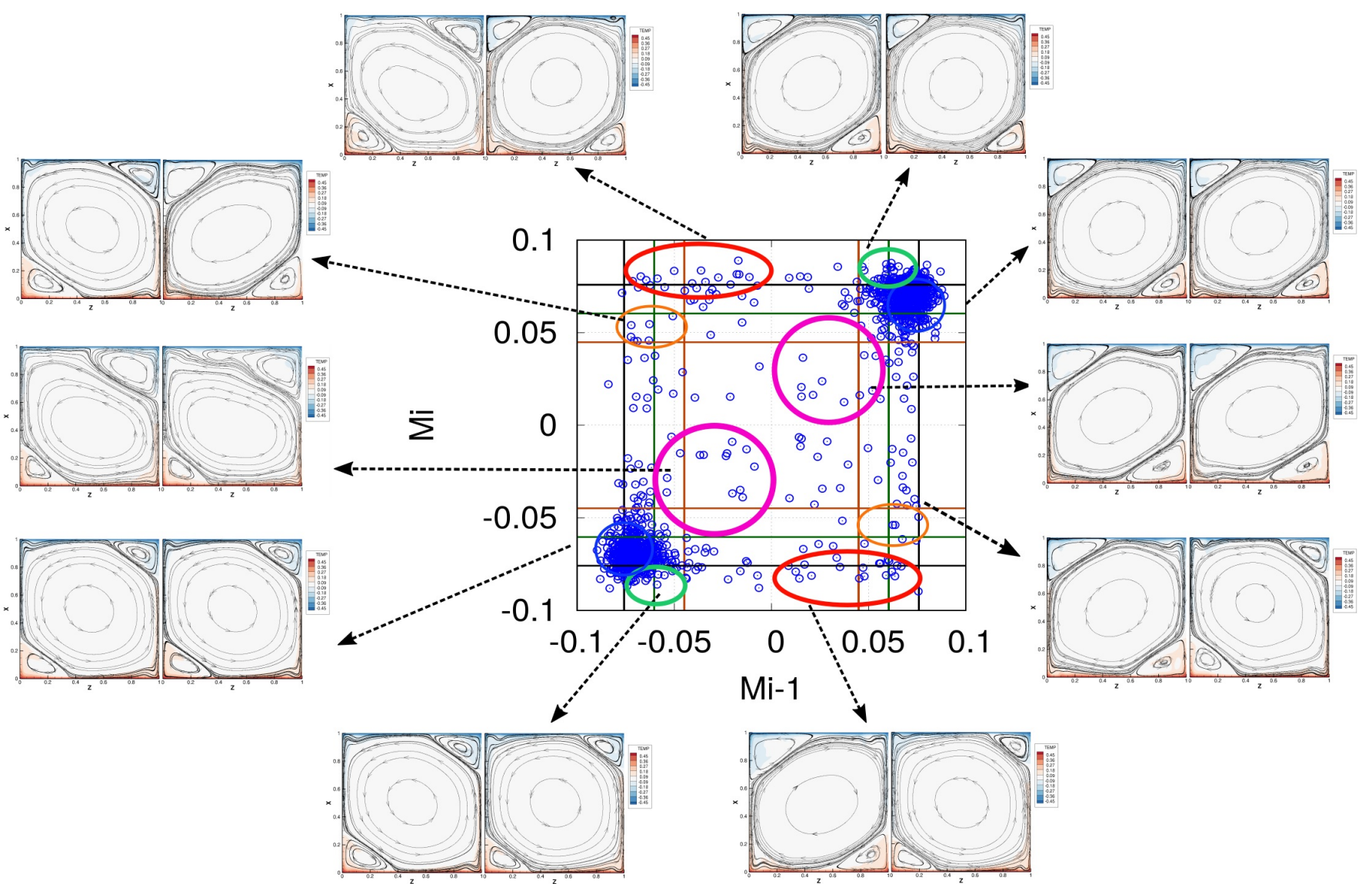

FIG. 19. Clusters in Rayleigh-Bénard attractors at $R a=3 \cdot 10^{7}$ and the related mean flow patterns. Same legend as in figure 16 
a)
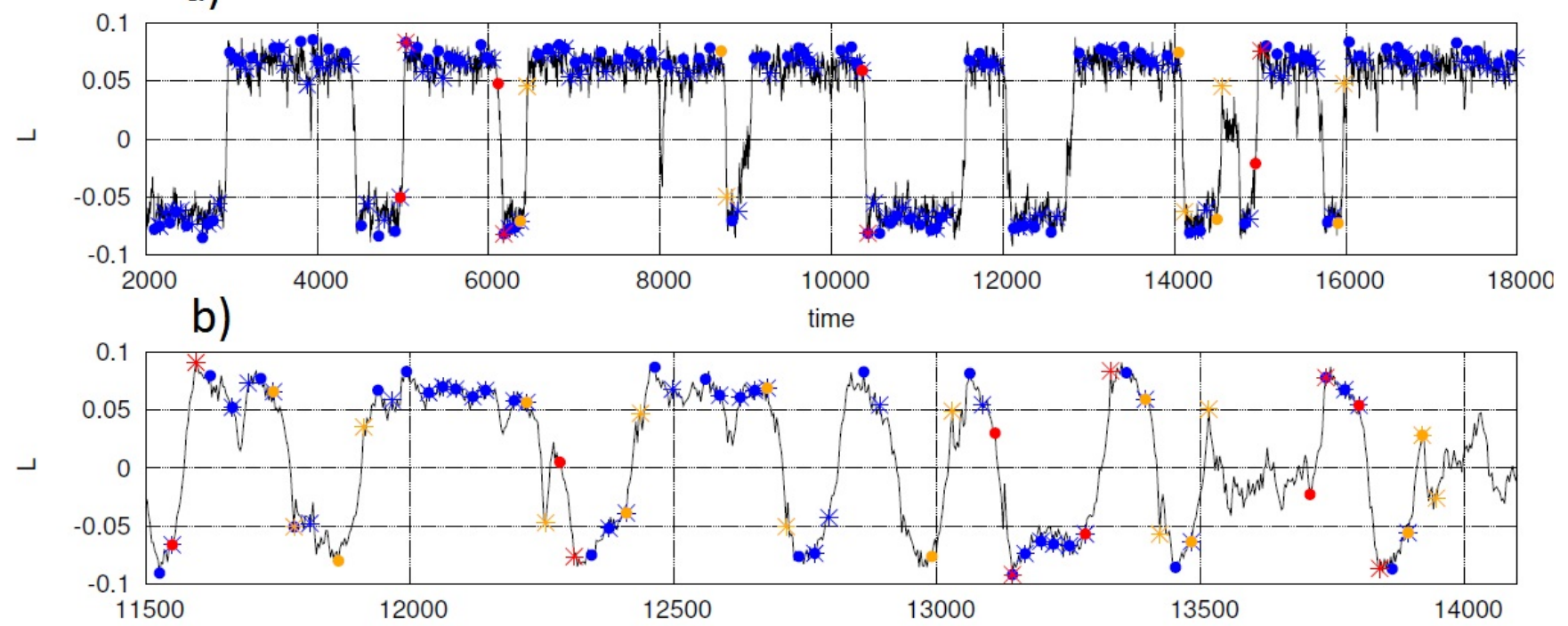

c)

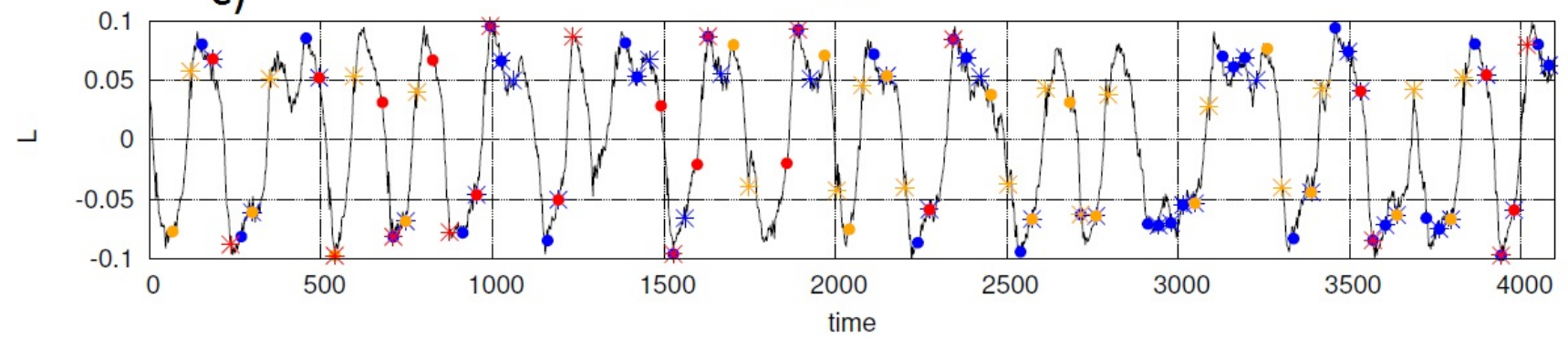

FIG. 20. Particular sequence of the angular momentum $L$ time series at $R a=3 \cdot 10^{7}$ (a), $R a=5 \cdot 10^{7}$ (b) and $R a=10^{8}$ (c). Markers give locations of all points contained inside the first part of release (orange), complete release (red) and accumulation (blue) clusters. $\bullet\left(M_{i-1}\right), *(M i)$ peaks. 